\title{
ELLIPTIC CORNER OPERATORS IN SPACES WITH CONTINUOUS RADIAL ASYMPTOTICS II
}

\author{
BOGDAN ZIEMIAN \\ Institute of Mathematics, Polish Academy of Sciences \\ Śniadeckich 8, 00-950 Warszawa, Poland
}

\begin{abstract}
Asymptotic expansions at the origin with respect to the radial variable are established for solutions to equations with smooth 2-dimensional singular Fuchsian type operators.
\end{abstract}

Introduction. This paper completes the results of paper [11] in which regularity of solutions to equations with elliptic corner operators (i.e. $n$-dimensional Fuchsian operators) is studied in the spaces $M(\Omega ; \varrho)$ of distributions with continuous radial asymptotics. Here we introduce subspaces $Z(\Omega ; \varrho)$ and $Z_{\mathrm{d}}(\Omega ; \varrho)$ of $M(\Omega ; \varrho)$ and prove a generalized Taylor formula for elements of those subspaces. This is preceded by preliminaries on the modified Cauchy transformation needed to establish a Mittag-Leffler type decomposition for holomorphic functions with a growth control along the imaginary axis. Then we study solutions to homogeneous equations $R\left(x_{1}, x_{2}, x_{1} \partial / \partial x_{1}, x_{2} \partial / \partial x_{2}\right) u=0$ with $R\left(x_{1}, x_{2}, \zeta_{1}, \zeta_{2}\right)$ an elliptic symbol, on proper cones in the positive quadrant in $\mathbb{R}^{2}$. The solutions $u$ are shown to belong to $Z_{\mathrm{d}}(\Omega ; \varrho)$.

In contrast to solutions to Fuchsian equations in the sense of BaouendiGoulaouic, the solutions $u$ to $R u=0$ do not expand in discrete powers of the radial variable. Instead, for $n=2$, we have "continuous" asymptotic expansions whose densities are distributions supported by several half lines parallel to the real axis. The densities are equal to the boundary values of the Mellin transforms of $u$ times the factor $(2 \pi i)^{-1}$. Moreover, they extend to holomorphic functions with logarithmic singularities situated in a discrete lattice in $\mathbb{C}$. This is resemblant of the resurgence phenomenon of Jean Ecalle and is further investigated in a forthcoming paper [12].

The paper ends with an explicit example covering the case of the operator $\widetilde{\Delta}=\left(x_{1} \partial / \partial x_{1}\right)^{2}+\left(x_{2} \partial / \partial x_{2}\right)^{2}$.

Some results of this paper appeared in [13]. The reader interested in a system- 
atic presentation of the theory of the Mellin transformation and Fuchsian PDEs is referred to the book [7].

Acknowledgements. Part of the work was carried out during the author's stay in Japan as a visiting professor at the University of Tokyo. The author thanks Prof. H. Komatsu for the invitation and Prof. A. Kaneko for his care and kind interest. Warm thanks are also due to Dr. G. Eysik for a critical reading of the manuscript.

1. Notation, definitions and preliminaries. Throughout the paper we use the following vector notation: if $a, b \in \mathbb{R}^{n}, a=\left(a_{1}, \ldots, a_{n}\right), b=\left(b_{1}, \ldots, b_{n}\right)$ then $a<b(a \leq b$, resp. $)$ denotes $a_{j}<b_{j}\left(a_{j} \leq b_{j}\right.$, resp. $)$ for $j=1, \ldots, n$. We write $\mathbb{R}_{+}^{n}=\left\{x \in \mathbb{R}^{n}: 0<x\right\}, \mathbb{R}_{-}=\{x \in \mathbb{R}: x<0\}, I=(0, t]=\left\{x \in \mathbb{R}_{+}^{n}: x \leq t\right\}$ where $t \in \mathbb{R}_{+}^{n}$. We also write $\mathbf{1}=(1, \ldots, 1) \in \mathbb{R}^{n}$.

$\mathbb{Z}$ is the set of integers and $\mathbb{N}_{0}$ the set of nonnegative integers. If $x \in \mathbb{R}_{+}^{n}$ and $z=\left(z_{1}, \ldots, z_{n}\right) \in \mathbb{C}^{n}$ we write $x^{z}=x_{1}^{z_{1}} \ldots x_{n}^{z_{n}}$. Vector notation is also used for differentiations. Namely we write

$$
\frac{\partial}{\partial x}=\left(\frac{\partial}{\partial x_{1}}, \ldots, \frac{\partial}{\partial x_{n}}\right), \quad x \frac{\partial}{\partial x}=\left(x_{1} \frac{\partial}{\partial x_{1}}, \ldots, x_{n} \frac{\partial}{\partial x_{n}}\right)
$$

and if $\nu \in \mathbb{N}_{0}^{n}$ then

$$
\left(\frac{\partial}{\partial x}\right)^{\nu}=\frac{\partial^{\nu_{1}}}{\partial x_{1}^{\nu_{1}}} \ldots \frac{\partial^{\nu_{n}}}{\partial x_{n}^{\nu_{n}}}, \quad\left(x \frac{\partial}{\partial x}\right)^{\nu}=\left(x_{1} \frac{\partial}{\partial x_{1}}\right)^{\nu_{1}} \ldots\left(x_{n} \frac{\partial}{\partial x_{n}}\right)^{\nu_{n}} .
$$

For points $a \in \mathbb{R}^{n}$ we write $a=\left(a_{1}, a^{\prime}\right)$ where $a_{1} \in \mathbb{R}, a^{\prime} \in \mathbb{R}^{n-1}$, and similarly for $\zeta \in \mathbb{C}^{n}, \zeta=\left(\zeta_{1}, \zeta^{\prime}\right), \zeta_{1} \in \mathbb{C}, \zeta^{\prime} \in \mathbb{C}^{n-1}$. We also consider sets $W \subset \mathbb{C}^{n}$ of the form $W=W^{1} \times W^{\prime}$ where $W^{1} \subset \mathbb{C}, W^{\prime} \subset \mathbb{C}^{n-1}$. For a set $W \subset \mathbb{C}^{n}$ and a vector $a \in \mathbb{R}^{n}$ we write $W+a=\left\{z \in \mathbb{C}^{n}: z-a \in W\right\}$.

For an open set $V \subset \mathbb{R}^{n}, C_{0}^{\infty}(V)$ is the space of compactly supported $C^{\infty}$ functions on $V, D^{\prime}(V)$ is the space of distributions on $V$.

$S\left(\mathbb{R}^{n}\right)$ denotes the Schwartz space of rapidly decreasing functions, $S^{\prime}\left(\mathbb{R}^{n}\right)$ is the space of tempered distributions. For a compact set $K \subset \mathbb{R}^{n}, S\left(\mathbb{R}^{n} \backslash K\right)=$ $\left\{\sigma \in \mathbb{C}^{\infty}\left(\mathbb{R}^{n}\right): \operatorname{supp} \sigma \subset \mathbb{R}^{n} \backslash K,\left|\|\sigma \mid\|_{p}<\infty\right.\right.$ for any $\left.p \in \mathbb{N}_{0}\right\}$ where

$$
\|\mid \sigma\|_{p}=\sup _{x \in \mathbb{R}^{n}}(1+\|x\|)^{p}\left(\sum_{|\alpha| \leq p}\left|D^{\alpha} \sigma(x)\right|\right) .
$$

For an open set $W \subset \mathbb{C}^{n}, \mathcal{O}(W)$ denotes the space of holomorphic functions on $W$ and if $K$ is a compact subset of $\mathbb{C}^{n}$ then $\mathcal{A}(K)=\lim _{W \supset K} \mathcal{O}(W)$. The dual space $\mathcal{A}^{\prime}(K)$ is called the space of analytic functionals with carrier $K$. By $B(\mathbb{R})=\mathcal{O}(\mathbb{C} \backslash \mathbb{R}) / \mathcal{O}(\mathbb{C})$ we denote the space of hyperfunctions on $\mathbb{R}$. An element $T \in B(\mathbb{R})$ is an equivalence class of a $\psi \in \mathcal{O}(\mathbb{C} \backslash \mathbb{R})$ modulo $\mathcal{O}(\mathbb{C})$ which we write as $T=[\psi]$ and verbalize as: $\psi$ is a defining function for $T$. The space $B_{K}(\mathbb{R})$ of hyperfunctions supported by a compact set $K \subset \mathbb{R}$ is isomorphic to $\mathcal{A}^{\prime}(K)$ : 
Theorem A (Martineau, Harvey [2]).

$$
B_{K}(\mathbb{R}) \cong \mathcal{A}^{\prime}(K) \text {. }
$$

The isomorphism is given by the duality

$$
\langle[\psi], \varphi\rangle=-\int_{\Gamma} \psi(z) \varphi(z) d z \quad \text { for } \varphi \in \mathcal{A}(K)
$$

where $[\psi] \in B_{K}(\mathbb{R})$ is a hyperfunction having $\psi \in \mathcal{O}(\mathbb{C} \backslash K)$ as a defining function and $\Gamma$ is a curve encircling $K$ once in the anti-clockwise direction and contained in the set where $\varphi$ is holomorphic.

We also recall the following characterization of distributions:

Theorem B (Painlevé [2]). Let $\psi \in \mathcal{O}(\mathbb{C} \backslash \mathbb{R})$ be of polynomial growth near $\mathbb{R}$ (i.e. $|\psi(a+i b)| \leq C|b|^{-p}$ for $b$ close to zero, with $C=C(a)>0, p=p(a) \in \mathbb{Z}$ locally bounded in $a \in \mathbb{R})$. Then in the sense of distributional convergence in $\mathbb{R}$ the limit

$$
b(\psi) \stackrel{\text { def }}{=} \lim _{b \rightarrow 0_{+}} \psi(\cdot+i b)-\lim _{b \rightarrow 0_{+}} \psi(\cdot-i b)
$$

exists and $b(\psi) \in D^{\prime}(\mathbb{R})$. Moreover, $b(\psi)=[\psi]$ under the inclusion $D^{\prime}(\mathbb{R}) \subset B(\mathbb{R})$ derived from the isomorphism of Theorem A.

We call $b(\psi)$ the boundary value or the jump of $\psi$ across $\mathbb{R}$.

Sometimes, it will be convenient to consider the above spaces transformed to an imaginary line $N \subset \mathbb{C}$ by means of a linear parametrization of $N$. More specifically, we consider the spaces $B(N) \cap S^{\prime}(N \backslash K)$ where $K$ is a compact subset of the imaginary line $N=r+i \mathbb{R}$ for some fixed $r \in \mathbb{R}$. Observe that if $K_{\varepsilon}=\{\zeta \in N: \operatorname{dist}(K, \zeta) \leq \varepsilon\}$ then

$$
B(N) \cap S^{\prime}(N \backslash K)=\bigcap_{\varepsilon>0}\left(\mathcal{A}^{\prime}\left(K_{\varepsilon}\right)+S^{\prime}(N)\right),
$$

where the sign "+" denotes the sum of analytic functionals.

More generally, $\mathcal{A}\left(\mathbb{R}^{n-1} ; S^{\prime}(\mathbb{R})\right)\left(\mathcal{O}\left(\mathbb{C}^{n-1} ; S^{\prime}(\mathbb{R})\right)\right.$, resp. $)$ denotes the space of analytic (holomorphic, resp.) functions on $\mathbb{R}^{n-1}\left(\mathbb{C}^{n-1}\right.$, resp.) with values in $S^{\prime}\left(\mathbb{R}^{n}\right)$, i.e. functions $\mathbb{R}^{n-1} \ni x \mapsto T(x) \in S^{\prime}(\mathbb{R})$ such that for any $\sigma \in S(\mathbb{R})$ the function $\mathbb{R}^{n-1} \ni x \mapsto T(x)[\sigma] \in \mathbb{C}$ is analytic, and similarly for the other case. We also make use of the isomorphism $S^{\prime}\left(\mathbb{R}^{n}\right) \simeq S^{\prime}\left(\mathbb{R}^{n-1} ; S^{\prime}(\mathbb{R})\right)$ where the right-hand side is the space of continuous linear mappings on $S\left(\mathbb{R}^{n-1}\right)$ with values in $S^{\prime}(\mathbb{R})$. Similarly, we use $S^{\prime}\left(\mathbb{R}^{n}\right) \simeq S^{\prime}\left(\mathbb{R} ; S^{\prime}\left(\mathbb{R}^{n-1}\right)\right)$. Both isomorphisms can be regarded as $S^{\prime}$ versions of the Schwartz kernel theorem ([1], [7]).

For the sake of completeness, below we briefly review the main facts on the Mellin transformation which are used in this paper (for details cf. [6], [7], [9]).

Fix $t \in \mathbb{R}_{+}^{n}$. Let $I=(0, t]$. We denote by $C^{\infty}(I)$ the set of complex functions on $I$ which are restrictions to $I$ of smooth functions on $\mathbb{R}_{+}^{n}$. 
Let $\alpha \in \mathbb{R}^{n}$. We denote by $M_{\alpha}=M_{\alpha}(I)$ the space of all $\varphi \in C^{\infty}(I)$ such that for every $\nu \in \mathbb{N}_{0}^{n}$

$$
\varrho_{\alpha, \nu}(\varphi)=\sup _{x \in I}\left|x^{\alpha+1}\left(x \frac{\partial}{\partial x}\right)^{\nu} \varphi(x)\right|
$$

is finite, with the topology given by the seminorms $\varrho_{\alpha, \nu}, \nu \in \mathbb{N}_{0}^{n}$. The space $M_{(\omega)}=M_{(\omega)}(I)$ for $\omega \in(\mathbb{R} \cup\{\infty\})^{n}$ is the inductive limit

$$
M_{(\omega)}=\underset{\alpha<\omega}{\bigcup} M_{\alpha}(I) .
$$

The space $M^{\prime}=\bigcup_{\omega \in \mathbb{R}^{n}} M_{(\omega)}^{\prime} \subset D^{\prime}\left(\mathbb{R}_{+}^{n}\right)$, where $M_{(\omega)}^{\prime}$ is the dual of $M_{(\omega)}$, is called the space of Mellin (transformable) distributions. We now define the Mellin transform of $u \in M_{(\omega)}^{\prime}$. Set

$$
\mathcal{M} u(z)=u\left[x^{-z-1}\right] \quad \text { for } z \in \mathbb{C}^{n}, \operatorname{Re} z<\omega .
$$

Then $\mathcal{M} u$ is holomorphic for $\operatorname{Re} z<\omega$. The Mellin transform of $u$ is any holomorphic extension of the function defined above.

We introduce a scale $M_{(\omega)}^{\prime s} \subset M_{(\omega)}^{\prime}$ for $s \in \mathbb{R}$ (see [10] and [4]) as follows: $u \in M_{(\omega)}^{\prime s}$ if for every $\alpha<\omega$ there exists a constant $C=C(\alpha)$ such that

$$
|\mathcal{M} u(\alpha+i \beta)| \leq C(1+\|\beta\|)^{s} \quad \text { for } \beta \in \mathbb{R}^{n} .
$$

The Mellin transformation introduced above satisfies the following operational identities. If $u \in M_{(\omega)}^{\prime}, a \in \mathbb{C}^{n}$ then

$$
\mathcal{M}\left(x^{a} u\right)(z)=\mathcal{M} u(z-a) \quad \text { for } \operatorname{Re} z<\omega+\operatorname{Re} a .
$$

If $\nu \in \mathbb{N}_{0}^{n},|\nu|=1$, then

$$
\mathcal{M}\left(\left(\frac{\partial}{\partial x}\right)^{\nu} u\right)(z)=\left(z^{\nu}+1\right) \mathcal{M} u(z+\nu) \quad \text { for } \operatorname{Re} z<\omega-\nu .
$$

Since we also consider distributions in $D^{\prime}\left(\mathbb{R}_{+}^{n}\right)$ with unbounded support we introduce the following spaces (see [6], Section 5).

Let $\alpha \in \mathbb{R}^{n}$ and let $\mu: \mathbb{R}^{n} \rightarrow \mathbb{R}_{+}^{n}$ be the exponential mapping $\mu(s)=$ $\left(e^{-s_{1}}, \ldots, e^{-s_{n}}\right)$. We define the space $\mathfrak{M}_{\alpha}^{\prime}=\mathfrak{M}_{\alpha}^{\prime}\left(\mathbb{R}_{+}^{n}\right)$ as the dual of the space

$$
\mathfrak{M}_{\alpha}=\mathfrak{M}_{\alpha}\left(\mathbb{R}_{+}^{n}\right)=\left\{\sigma \in C^{\infty}\left(\mathbb{R}_{+}^{n}\right):\left(x^{\alpha+1} \sigma\right) \circ \mu \in S\left(\mathbb{R}^{n}\right)\right\}
$$

with the natural topology induced from $S\left(\mathbb{R}^{n}\right)$. Note that $u \in \mathfrak{M}_{\alpha}^{\prime}$ if and only if $e^{\alpha s}(u \circ \mu) \in S^{\prime}\left(\mathbb{R}^{n}\right)$

The Mellin $\mathcal{M}_{\alpha}$ transform of $u \in \mathfrak{M}_{\alpha}^{\prime}$ is defined as

$$
\mathcal{M}_{\alpha} u=(2 \pi)^{n} \mathcal{F}^{-1}\left(e^{\alpha s}(u \circ \mu)\right) \in S^{\prime}\left(\mathbb{R}^{n}\right)
$$

where the inverse Fourier transform $\mathcal{F}^{-1}$ is defined as

$$
\mathcal{F}^{-1} \psi(b)=\frac{1}{(2 \pi)^{n}} \int_{\mathbb{R}^{n}} e^{i s b} \psi(s) d s \quad \text { for } \psi \in S\left(\mathbb{R}^{n}\right) .
$$


$\mathcal{M}_{\alpha}$ is compatible with $\mathcal{M}$ in the sense that if $u \in M_{(\omega)}(I)$ then for every $\alpha<\omega$, we have $u \in \mathfrak{M}_{\alpha}^{\prime}$, the tempered distribution $\mathcal{M}_{\alpha} u$ is actually a function, and $\mathcal{M}_{\alpha} u(\beta)=\mathcal{M} u(\alpha+i \beta)$ for $\beta \in \mathbb{R}^{n}$.

Restrictions of distributions in $\mathfrak{M}_{\alpha}^{\prime}$ to compact sets are in $M_{(\alpha)}^{\prime}$. More precisely, if $u \in \mathfrak{M}_{\alpha}^{\prime}$ then $\varphi u \in M_{(\alpha)}^{\prime}(I)$ for every $\varphi \in C_{0}^{\infty}\left(\mathbb{R}^{n}\right)$ with supp $\varphi \cap \mathbb{R}_{+}^{n} \subset I$.

To recall the definition of the space $M(\Omega ; \varrho)$ of distributions with continuous radial asymptotics we need some additional notation:

Let $A: \mathbb{C}^{n} \rightarrow \mathbb{C}^{n}$ be the linear transformation defined by $A z=\zeta$ where

$$
\begin{gathered}
\zeta_{1}=z_{1}+\ldots+z_{n}, \\
\zeta_{j}=z_{j} \quad \text { for } j=2, \ldots, n .
\end{gathered}
$$

The formula

$$
(\mathcal{M} u) \circ A^{-1}(\zeta)=\mathcal{M}(u \circ S)(\zeta)
$$

for $u \in M^{\prime}, \operatorname{supp} u \subset \Gamma$, a proper cone in $\mathbb{R}_{+}^{n}$ (i.e. $\bar{\Gamma} \cap \overline{\mathbb{R}_{+}^{n}}=\{0\}$ where overbar denotes closure in $\mathbb{R}^{n}$ ), relates this transformation (see Proposition 1 in [11]) to the passage to the "radial" coordinates $x=S(y)$, with $S: \mathbb{R}_{+}^{n} \rightarrow \mathbb{R}_{+}^{n}$, given by

$$
x_{1}=y_{1}, \quad x_{j}=y_{1} y_{j} \quad \text { for } j=2, \ldots, n .
$$

Definition A (see Def. 1 in [11]). Let $\Omega^{1}$ be an $\mathbb{R}_{-}$-connected open subset of $\mathbb{C}$, i.e. a subset which together with any point $\dot{\zeta}_{1} \in \Omega^{1}$ contains the half-line $\dot{\zeta}_{1}+\overline{\mathbb{R}_{-}}$. Also suppose that for any $r \in \operatorname{Re} \Omega^{1} \stackrel{\text { def }}{=}\left\{\operatorname{Re} \zeta_{1}: \zeta_{1} \in \Omega^{1}\right\}$ the set $\Lambda_{r}=\left\{\zeta_{1} \in \mathbb{C} \backslash \Omega^{1}: \operatorname{Re} \zeta_{1} \leq r\right\}$ is compact in $\mathbb{C}$. Let $\varrho: \operatorname{Re} \Omega^{1} \rightarrow \mathbb{R}$ be a nondecreasing function. We say that a Mellin distribution $u$ supported by a proper cone $\Gamma$ belongs to $M(\Omega ; \varrho)$ where

$$
\Omega=A^{-1}\left(\Omega^{1} \times \mathbb{C}^{n-1}\right)
$$

if the function $H=\mathcal{M} u \circ A^{-1}$ satisfies the following conditions:

(i) $H \in \mathcal{O}\left(\Omega^{1} \times \mathbb{C}^{n-1}\right)$.

(ii) For any open neighbourhood $W$ of $\Lambda=\mathbb{C} \backslash \Omega^{1}$

$$
|H(a+i b)| \leq C(1+\|b\|)^{\varrho\left(a_{1}\right)} \quad \text { for } a+i b \in(\mathbb{C} \backslash W) \times \mathbb{C}^{n-1}
$$

where $C=C(W, a)$ is locally bounded in $a \in \operatorname{Re}(\mathbb{C} \backslash W) \times \mathbb{R}^{n-1}$.

Definition B. Let $\delta \dot{x} \in \mathbb{R}_{+}^{n}$. A conical cut-off function at $(0, \delta \dot{x})$ is any function $\kappa \in C^{\infty}\left(\mathbb{R}_{+}^{n}\right)$ of the form

$$
\kappa=\varphi \cdot \widetilde{\kappa}
$$

where $\varphi \in C_{0}^{\infty}\left(\mathbb{R}_{+}^{n}\right), \varphi \equiv 1$ in a neighbourhood of zero, $\widetilde{\kappa} \in C^{\infty}\left(\mathbb{R}_{+}^{n}\right)$ is homogeneous of order zero, $\widetilde{\kappa}(\delta \dot{x}) \neq 0$ and $\widetilde{\kappa}$ is supported in a proper cone in $\mathbb{R}_{+}^{n}$.

Finally, for a compact set $K \subset \mathbb{C}$ and a constant function $\varrho(r) \equiv s \in \mathbb{R}$ we denote by $\mathcal{A}^{\prime}\left(K ; M\left(\mathbb{C}^{n-1} ; s\right)\right)$ the space of $M\left(\mathbb{C}^{n-1} ; s\right)$-valued continuous analytic 
functionals $T$ with carrier $K$ such that for any compact $U \subset C$ with $K \subset \operatorname{Int} U$

$$
\left|\mathcal{M}^{\prime} T[\varphi]\left(a^{\prime}+i b^{\prime}\right)\right| \leq C\left(1+\left\|b^{\prime}\right\|\right)^{s} \sup _{\theta \in U}|\varphi(\theta)| \quad \text { for } \varphi \in \mathcal{A}(U) \text { and } b^{\prime} \in \mathbb{R}^{n-1}
$$

locally uniformly in $a^{\prime} \in \mathbb{R}^{n-1}$. Here $\mathcal{M}^{\prime}$ is the partial Mellin transform in the variables $\zeta^{\prime} \in \mathbb{C}^{n-1}$. Similarly, $T \in D^{\prime}\left(\mathbb{R} ; M\left(\mathbb{C}^{n-1} ; s\right)\right)$ if for any compact $K \subset \mathbb{R}$

$$
\left|\mathcal{M}^{\prime} T[\varphi]\left(a^{\prime}+i b^{\prime}\right)\right| \leq C\left(1+\left\|b^{\prime}\right\|\right)^{s} \sum_{|\alpha| \leq m} \sup _{b_{1} \in \mathbb{R}}\left|\left(\frac{\partial}{\partial b_{1}}\right)^{\alpha} \varphi\left(b_{1}\right)\right| \quad \text { for } b^{\prime} \in \mathbb{R}^{n-1},
$$

$\varphi \in C_{0}^{\infty}(\mathbb{R}), \operatorname{supp} \varphi \subset K$, with $C=C\left(K, a^{\prime}\right), m=m\left(K, a^{\prime}\right)$ locally bounded in $a^{\prime} \in \mathbb{R}^{n-1}$.

2. The spaces $Z(\Omega ; \varrho), Z_{\mathrm{d}}(\Omega ; s)$. We introduce subspaces $Z(\Omega ; \varrho)$ and $Z_{\mathrm{d}}(\Omega ; s)$ of $M(\Omega ; \varrho)$ which contain information on the behaviour of Mellin transforms near the boundary of $\Omega$.

Definition 1. Let $\Omega$ and $\varrho$ be as in Definition A. We say that a Mellin distribution $u$ with support in a proper cone $\Gamma \subset \mathbb{R}_{+}^{n}$ belongs to $Z(\Omega ; \varrho)$ if the function $H(\zeta)=\mathcal{M} u \circ A^{-1}(\zeta)$ satisfies the following conditions for all $r \in \operatorname{Re} \Omega^{1}$ :

(i) $H \in \mathcal{O}\left(\Omega^{1} \times \mathbb{C}^{n-1}\right)$.

(ii) For any open neighbourhood $W$ of $\Lambda=\mathbb{C} \backslash \Omega^{1}$

$$
|H(a+i b)| \leq C(1+\|b\|)^{\varrho\left(a_{1}\right)} \quad \text { for } a+i b \in(\mathbb{C} \backslash W) \times \mathbb{C}^{n-1}
$$

where $C=C(W, a)$ is locally bounded with respect to $a \in \operatorname{Re} \Omega^{1} \times \mathbb{R}^{n-1}$.

(iii) There exists a $B(r+i \mathbb{R}) \cap S^{\prime}\left((r+i \mathbb{R}) \backslash K^{r}\right)$-valued holomorphic function

$$
\mathbb{C}^{n-1} \ni \zeta^{\prime} \mapsto H_{r, \zeta^{\prime}} \in B(r+i \mathbb{R}) \cap S^{\prime}\left((r+i \mathbb{R}) \backslash K^{r}\right)
$$

where $K^{r}=\Lambda \cap\left\{\operatorname{Re} \zeta_{1}=r\right\}$, which is an extension of the function

$$
(r+i \mathbb{R}) \backslash K^{r} \ni \zeta_{1} \mapsto H\left(\zeta_{1}, \zeta^{\prime}\right) .
$$

(iv) For every $0<\varepsilon<\stackrel{\circ}{\varepsilon}$, if

$$
\chi_{\varepsilon}\left(\zeta_{1}\right)= \begin{cases}1 & \text { for } \zeta_{1} \in K_{\varepsilon}^{r}, \\ 0 & \text { for } \zeta_{1} \in(r+i \mathbb{R}) \backslash K_{\varepsilon}^{r},\end{cases}
$$

where $K_{\varepsilon}^{r}=\left\{\zeta_{1} \in r+i \mathbb{R}: \operatorname{dist}\left(\zeta_{1}, K^{r}\right) \leq \varepsilon\right\}$, then

$$
\left|\chi_{\varepsilon} H_{r, \zeta^{\prime}}[\varphi]\right| \leq C\left(1+\left\|b^{\prime}\right\|\right)^{\varrho(r)} \sup _{\theta \in V}|\varphi(\theta)| \quad \text { for } b^{\prime} \in \mathbb{R}^{n-1}
$$

(note that $H_{r, \zeta^{\prime}}$ is analytic outside $K^{r}$ and hence $\chi_{\varepsilon} H_{r, \zeta^{\prime}}$ makes sense) where $V \subset \mathbb{C}$ is any compact set such that $K_{\varepsilon}^{r} \subset \operatorname{Int} V, \varphi \in \mathcal{A}(V)$, and $C=C\left(V, \varepsilon, a^{\prime}\right)$ is locally bounded in $a^{\prime} \in \mathbb{R}^{n-1}$.

Next we define a subspace of $Z(\Omega ; \varrho)$ which appears frequently in applications and is much easier to deal with. We consider a special case of $\Omega^{1}=\mathbb{C} \backslash \bigcup_{j=1}^{k} L_{j}$ where $L_{j}$ are closed half lines parallel to the real axis. We write $\mathbf{B}=\left\{B_{1}, \ldots, B_{k}\right\}$ where $B_{j}=\operatorname{Im} L_{j}$. 
Definition 2. Let $\Omega=A^{-1}\left(\Omega^{1} \times \mathbb{C}^{n-1}\right)$ with $\Omega^{1}$ as above, and $\varrho \equiv s \in \mathbb{R}$ a constant. We write $u \in Z_{\mathrm{d}}(\Omega ; s)$ if $u$ is a Mellin distribution supported by a proper cone $\Gamma \subset \mathbb{R}_{+}^{n}$ and the function $H(\zeta)=\mathcal{M} u \circ A^{-1}(\zeta)$ satisfies the following conditions:

(i) $H \in \mathcal{O}\left(\Omega^{1} \times \mathbb{C}^{n-1}\right)$.

(ii) For every open neighbourhood $W$ of $\bigcup_{j=1}^{k} L_{j}$

$$
|H(a+i b)| \leq C(1+\|b\|)^{s} \quad \text { for } a+i b \in(\mathbb{C} \backslash W) \times \mathbb{C}^{n-1}
$$

locally uniformly with respect to $a \in \mathbb{R}^{n}$.

(iii) $|H(a+i b)| \leq \widetilde{C}\left(1+\left\|b^{\prime}\right\|\right)^{s} /\left(\operatorname{dist}\left(b_{1}, \mathbf{B}\right)\right)^{m}$ for $b_{1}$ close to $\mathbf{B}$ and $a^{\prime}+i b^{\prime} \in$ $\mathbb{C}^{n-1}$, for some constants $0<\widetilde{C}=\widetilde{C}(a), m=m(a) \in \mathbb{R}$ locally bounded for $a \in \mathbb{R}^{n}$.

We also set $Z_{\mathrm{d}}(\Omega ;-\infty)=\bigcap_{s \in \mathbb{R}} Z_{\mathrm{d}}(\Omega ; s)$.

To see that $Z_{\mathrm{d}}(\Omega ; s) \subset Z(\Omega ; s)$ we note the following simple

Lemma 1. If $H \in \mathcal{O}\left(\Omega^{1} \times \mathbb{C}^{n-1}\right)$ satisfies (ii) and (iii) of Definition 2 then there exists a holomorphic $S^{\prime}$-valued function

$$
\mathbb{C}^{n-1} \ni \zeta^{\prime} \mapsto H_{r, \zeta^{\prime}} \in S^{\prime}(r+i \mathbb{R})
$$

extending the function

$$
r+i(\mathbb{R} \backslash \mathbf{B}) \ni \zeta_{1} \mapsto H\left(\zeta_{1}, \zeta^{\prime}\right)
$$

and such that for any $\varphi \in C_{0}^{\infty}(r+i \mathbb{R})$ with support in a compact set $E \subset r+i \mathbb{R}$

$$
\left|H_{r, \zeta^{\prime}}[\varphi]\right| \leq C\left(1+\left\|b^{\prime}\right\|\right)^{s}\left(\sum_{j=0}^{m+1} \sup _{\zeta_{1} \in E}\left|\left(\frac{\partial}{\partial b_{1}}\right)\right|^{j} \varphi\left(\zeta_{1}\right)\right)
$$

with a constant $C=C(E)$ independent of $\zeta^{\prime} \in \mathbb{C}^{n-1}$.

Proof. The proof is standard and the desired extension is constructed by means of the $m+1$-th order primitive of the function $b_{1} \mapsto H\left(r+i b_{1}, \zeta^{\prime}\right)$ near the points of $\mathbf{B}$.

3. Modified Cauchy transformation in dimension 1. In this section we present in a slightly generalized setting certain results of Section 1 of [10]. The reader is directed to [10] for a more detailed exposition.

Let $\chi \in \mathbb{C}_{0}^{\infty}(\mathbb{R})$ with $\chi \equiv 1$ on $\left(0, t_{0}\right]$. A modified Cauchy kernel (determined by $\chi$ ) is the function

$$
G(z)=\mathcal{M} \chi(z) .
$$

It has the following properties (see Proposition 7.5 of [7]):

(i) $G \in \mathcal{O}(\mathbb{C} \backslash\{0\})$,

(ii) $G(z)=-1 / z+\widetilde{G}(z)$ with $\widetilde{G} \in \mathcal{O}(\mathbb{C})$, 
(iii) the function $\mathbb{R} \ni \beta \mapsto(\alpha+i \beta) G(\alpha+i \beta)$ is in $S(\mathbb{R})$ locally uniformly with respect to $\alpha \in \mathbb{R}$,

(iv) for every $\varepsilon>0$ and $m>0$ there exists a constant $C=C(\varepsilon, m)$ such that

$$
|G(\alpha+i \beta)| \leq C t_{0}^{-\alpha}(1+|\beta|)^{-m} \quad \text { for } \alpha>\varepsilon .
$$

Let $r \in \mathbb{R}$, let $K \subset r+i \mathbb{R}$ be a compact set and define $N=r+i \mathbb{R}$.

Definition 3. Let $T \in B(N) \cap S^{\prime}(N \backslash K)$. The functions

$$
\mathcal{C}^{ \pm} T(\zeta)=\frac{1}{2 \pi i} T[G(\zeta-\theta)] \quad \text { for } \pm \operatorname{Re} \zeta> \pm r
$$

are called the right and the left (modified) Cauchy transforms of $T$.

Note that, since for every $\zeta$ with $\operatorname{Re} \zeta \neq r$ the function $r+i \mathbb{R} \ni \theta \mapsto G(\zeta-\theta)$ is analytic and rapidly decreasing, $T[G(\zeta-\theta)]$ is well-defined and is a holomorphic function of $\zeta$.

Theorem 1. Let $T \in B(N) \cap S^{\prime}(N \backslash K)$. Then under the isomorphism of Theorems $\mathrm{A}$ and $\mathrm{B}$

$$
T=\left[\mathcal{C}^{-} T, \mathcal{C}^{+} T\right]
$$

where the right-hand side is a Fourier hyperfunction on $N$, with a defining function

$$
\Psi(\zeta)= \begin{cases}\mathcal{C}^{-} T(\zeta) & \text { for } \operatorname{Re} \zeta<r \\ \mathcal{C}^{+} T(\zeta) & \text { for } \operatorname{Re} \zeta>r\end{cases}
$$

Proof. By (2) write $T=T_{1}+T_{2}$ where $T_{1} \in \mathcal{A}^{\prime}\left(K_{\varepsilon}\right)$ (for some $\varepsilon>0$ ) and $T_{2} \in S^{\prime}(N)$. For $T_{2}$ the theorem coincides with Theorem 1 of [10]. In the case of $T_{1}$ we define for $\zeta \notin K_{\varepsilon}$

In view of (ii) we have

$$
\Psi_{1}^{G}(\zeta)=\frac{1}{2 \pi i} T_{1}[G(\zeta-\theta)]
$$

$$
\Psi_{1}^{G}(\zeta)=\frac{-1}{2 \pi i} T_{1}\left[\frac{1}{\zeta-\theta}\right]+\frac{1}{2 \pi i} T_{1}[\widetilde{G}(\zeta-\theta)] .
$$

Observe that the first summand is a standard defining function of $T_{1}$ and the second is entire on $\mathbb{C}$. Hence $\left[\Psi_{1}^{G}\right]=T_{1}$, which ends the proof.

4. Mittag-Leffler decomposition. Before stating the version of the MittagLeffler theorem which we need we prepare a suitable notation: Let $H \in \mathcal{O}\left(\Omega^{1}\right)$ where $\Omega^{1}$ is as in Definition A. Fix $r \in \operatorname{Re} \Omega^{1}$ and suppose that for some $\widetilde{m} \in \mathbb{N}_{0}$ and $t_{1} \in \mathbb{R}_{+}$

(4) $\left|H\left(a_{1}+i b_{1}\right)\right| \leq C t_{1}^{-a_{1}}\left(1+\left|b_{1}\right|\right)^{\tilde{m}} \quad$ for $a_{1}+i b_{1} \in \mathbb{C} \backslash W, a_{1} \leq r$, with $C=C(W)$, where $W$ is an open neighbourhood of $\Lambda=\mathbb{C} \backslash \Omega^{1}$. Denote by $\widetilde{H}_{r}$ an extension to $B(r+i \mathbb{R}) \cap S^{\prime}\left((r+i \mathbb{R}) \backslash K^{r}\right)$ of the function

$$
(r+i \mathbb{R}) \backslash K^{r} \ni \zeta_{1} \mapsto H\left(\zeta_{1}\right)
$$


where $K^{r}=\Lambda \cap\left\{\operatorname{Re} \zeta_{1}=r\right\}$. As usual, set $K_{\varepsilon}^{r}=\left\{r+i b_{1}: \operatorname{dist}\left(r+i b_{1}, K^{r}\right) \leq \varepsilon\right\}$ and

$$
\Lambda_{r}=\Lambda \cap\left\{\operatorname{Re} \zeta_{1} \leq r\right\} .
$$

Let $\Gamma$ be a smooth curve in $\Omega^{1}$ with end points at the end points of $K_{\varepsilon}^{r}$, having $\Lambda_{r}$ on its right. Define an analytic functional $\Xi_{r} \in \mathcal{A}^{\prime}\left(\Lambda_{r}\right)$ by

$$
\Xi_{r}[\varphi]=-\chi_{\varepsilon} \widetilde{H}_{r}[\varphi]-\int_{\Gamma} H(\theta) \varphi(\theta) d \theta \quad \text { for } \varphi \in \mathcal{A}\left(\Lambda_{r}\right)
$$

where $\chi_{\varepsilon}$ is defined as in Definition 1(iv).

Theorem 2. Assume that $t_{0}>t_{1}$ (see the beginning of Section 3 and (4)). Define

$$
\begin{array}{rlrl}
\Psi^{G}\left(\zeta_{1}\right) & =\frac{1}{2 \pi i} \Xi_{r}\left[G\left(\zeta_{1}-\theta\right)\right] & \text { for } \zeta_{1} \in \mathbb{C} \backslash \Lambda_{r}, \\
\mathcal{C}^{-} H\left(\zeta_{1}\right) & =\frac{1}{2 \pi i} \widetilde{H}_{r}\left[G\left(\zeta_{1}-\theta\right)\right] & & \text { for } \operatorname{Re} \zeta_{1}<r .
\end{array}
$$

Then $\Psi^{G} \in \mathcal{O}\left(\mathbb{C} \backslash \Lambda_{r}\right), \mathcal{C}^{-} H$ extends to a holomorphic function on $\Omega^{1} \cup\left\{\operatorname{Re} \zeta_{1}<r\right\}$ and

$$
H\left(\zeta_{1}\right)=\mathcal{C}^{-} H\left(\zeta_{1}\right)+\Psi^{G}\left(\zeta_{1}\right) \quad \text { for } \zeta_{1} \in \Omega^{1} .
$$

Proof. It is clear that $\Psi^{G} \in \mathcal{O}\left(\mathbb{C} \backslash \Lambda_{r}\right)$, while Theorem 1 implies that $\mathcal{C}^{-} H$ extends holomorphically to $\Omega^{1} \cup\left\{\operatorname{Re} \zeta_{1}<r\right\}$. We shall prove that (5) holds for $\zeta_{1} \in \Omega_{r}^{1}=\Omega^{1} \cap\left\{\operatorname{Re} \zeta_{1}<r\right\}$. To this end take $\dot{\zeta}_{1} \in \Omega_{r}^{1}$ and a curve $\Gamma^{1}$ consisting of the intervals $(r+i \mathbb{R}) \backslash K_{\varepsilon}^{r}$ and of a curve $\Gamma$ such that $\stackrel{\circ}{\zeta}_{1}$ remains to the left of $\Gamma^{1}$. Then

$$
\mathcal{C}^{-} H\left(\stackrel{\circ}{1}_{1}\right)+\Psi^{G}\left(\stackrel{\circ}{\zeta}_{1}\right)=\frac{1}{2 \pi i} \int_{\Gamma^{1}} H(\theta) G\left(\stackrel{\circ}{1}_{1}-\theta\right) d \theta
$$

Since $H$ is polynomially bounded in $\operatorname{Im} \theta$ and $G$ is rapidly decreasing $\operatorname{in} \operatorname{Im} \theta$ locally uniformly in $\operatorname{Re} \theta$, it follows from the residue theorem that for any $\widetilde{a}$ sufficiently large negative

$$
\frac{1}{2 \pi i} \int_{\Gamma^{1}} H(\theta) G\left(\stackrel{\circ}{\zeta}_{1}-\theta\right) d \theta=H\left(\stackrel{\circ}{\zeta}_{1}\right)+\frac{1}{2 \pi i} \int_{\operatorname{Re} \theta=\tilde{a}} H(\theta) G\left(\stackrel{\circ}{\zeta}_{1}-\theta\right) d \theta .
$$

Now it follows from (4), (iv) and easy estimates that

$$
\left|\int_{\operatorname{Re} \theta=\tilde{a}} H(\theta) G\left(\stackrel{\circ}{\zeta}_{1}-\theta\right) d \theta\right| \leq C t_{1}^{-\tilde{a}} t_{0}^{-\operatorname{Re} \stackrel{\circ}{\zeta}+\tilde{a}}=\widetilde{C}\left(\frac{t_{1}}{t_{0}}\right)^{-\tilde{a}} .
$$

Since we assumed that $t_{1}<t_{0}$ we see that the integral can be made arbitrarily small by letting $\widetilde{a} \rightarrow-\infty$. This ends the proof.

5. Generalized Taylor formula for distributions in $Z(\Omega ; \varrho)$. Now we are back in $\mathbb{C}^{n}$ and consider holomorphic functions $H$ satisfying (i)-(iv) of Defi- 
nition 1 . We define the left Cauchy transform $\mathcal{C}_{\zeta^{\prime}}^{-}\left(\zeta_{1}\right)$ by

$$
\mathcal{C}_{\zeta^{\prime}}^{-}\left(\zeta_{1}\right)=\frac{1}{2 \pi i} H_{r, \zeta^{\prime}}\left[G\left(\zeta_{1}-\theta\right)\right] \quad \text { for } \operatorname{Re} \zeta_{1}<r
$$

where $\zeta^{\prime} \in \mathbb{C}^{n-1}$ is a holomorphic parameter.

Lemma 2. Suppose $H \in \mathcal{O}\left(\Omega^{1} \times \mathbb{C}^{n-1}\right)$ satisfies (ii)-(iv) of Definition 1 for a fixed $a_{1}=r \in \operatorname{Re} \Omega^{1}$. Then

$$
\left|\mathcal{C}_{a^{\prime}+i b^{\prime}}^{-}\left(a_{1}+i b_{1}\right)\right| \leq C(1+\|b\|)^{\varrho(r)} \quad \text { for } a_{1}<r, a^{\prime}+i b^{\prime} \in \mathbb{C}^{n-1},
$$

locally uniformly in $a=\left(a_{1}, a^{\prime}\right)$ for $a_{1}<r, a^{\prime} \in \mathbb{R}^{n-1}$.

We omit the proof since it does not differ essentially from that of Lemma 2 in $[11]$.

Lemma 3. Let $\Xi \in \mathcal{A}^{\prime}(K)$ where $K \subset \mathbb{C}$ is compact. If

$$
\Psi^{G}\left(\zeta_{1}\right)=\frac{1}{2 \pi i} \Xi\left[G\left(\zeta_{1}-\theta\right)\right] \quad \text { for } \zeta_{1} \in \mathbb{C} \backslash K
$$

then

$$
\mathcal{M}\left(\Xi\left[y^{\theta} \chi(y)\right]\right)\left(\zeta_{1}\right)=2 \pi i \Psi^{G}\left(\zeta_{1}\right)
$$

Proof. In view of the isomorphism of Theorem A (see the proof of Theorem A), $\Xi$ can be represented as

$$
\Xi[\varphi]=-\int_{\Gamma} \Psi(\theta) \varphi(\theta) d \theta \quad \text { for } \varphi \in \mathcal{A}(K)
$$

where $\Gamma$ is a curve encircling $K$ once in the anti-clockwise direction contained in the set of holomorphy of $\varphi$, and $\Psi \in \mathcal{O}(\mathbb{C} \backslash K)$. We thus have

$$
\begin{aligned}
\mathcal{M}\left(\Xi\left[y^{\theta} \chi(y)\right]\right)\left(\zeta_{1}\right) & =-\int_{\Gamma} \Psi(\theta) \mathcal{M}\left(y^{\theta} \chi(y)\right)\left(\zeta_{1}\right) d \theta \\
& =-\int_{\Gamma} \Psi(\theta) G\left(\zeta_{1}-\theta\right) d \theta=2 \pi i \Psi^{G}\left(\zeta_{1}\right)
\end{aligned}
$$

since $\mathcal{M}\left(y^{\theta} \chi(y)\right)\left(\zeta_{1}\right)=G\left(\zeta_{1}-\theta\right)$.

THEOREM 3. A Mellin distribution $u$ belongs to $Z(\Omega ; \varrho)$ if and only if for any $r \in \operatorname{Re} \Omega^{1}$ there exist $T_{r} \in \mathcal{A}^{\prime}\left(\Lambda_{r} ; M\left(\mathbb{C}^{n-1} ; \varrho(r)\right)\right)$ and a distribution $R_{r} \in$ $M\left(\Omega_{r} ; \max (\varrho, \varrho(r))\right)$ where $\Omega_{r}=A^{-1}\left(\left(\Omega^{1} \cup\left\{\operatorname{Re} \zeta_{1}<r\right\}\right) \times \mathbb{C}^{n-1}\right)$ such that

$$
u \circ S=T_{r} \circ S^{\prime}\left[y_{1}^{\theta} \chi\left(y_{1}\right)\right]+R_{r} \circ S \quad\left(S \text { and } S^{\prime} \text { are defined by }(3)\right)
$$

where $\chi$ is in $C_{0}^{\infty}(\mathbb{R}), \chi \equiv 1$ in a neighbourhood of zero and for every fixed $y_{1}>0$, $y_{1}^{\theta}$ denotes the test function $\mathbb{C} \ni \theta \mapsto y_{1}^{\theta} \in \mathbb{C}$ (note that $T_{r}$ is regarded as an analytic functional in the variable $\theta)$. The $T_{r}$ is unique up to an $M\left(\mathbb{C}^{n-1} ; \varrho(r)\right)$ valued analytic functional with carrier in $K_{r}=\Lambda_{r} \cap\left\{\operatorname{Re} \zeta_{1}=r\right\}$. 
Proof. Let $u \in Z(\Omega ; \varrho)$ and $H(\zeta)=\mathcal{M} u \circ A^{-1}(\zeta)$. Then by Proposition 1 of [11], $u \circ S$ is a Mellin distribution supported by a cube $(0, \tilde{t}]$ for some $\tilde{t} \in \mathbb{R}_{+}^{n}$ (actually by $\left(0, \tilde{t}_{1}\right] \times\left[\widetilde{t}^{\prime}, \tilde{t}^{\prime}\right]$ for some ${\widetilde{t^{\prime}}}^{\prime} \in \mathbb{R}_{+}^{n-1})$ and

$$
H(\zeta)=\mathcal{M}(u \circ S)(\zeta) .
$$

Choose $r \in \operatorname{Re} \Omega^{1}$ and let $\mathbb{C}^{n-1} \ni \zeta^{\prime} \mapsto H_{r, \zeta^{\prime}}$ be the $B(r+i \mathbb{R}) \cap S^{\prime}((r+i \mathbb{R}) \backslash$ $K^{r}$ )-valued holomorphic function given by Definition 1(iii). Define a holomorphic family of analytic functionals $\Xi_{r, \zeta^{\prime}}$ as in Section 4 :

$$
\Xi_{r, \zeta^{\prime}}[\varphi]=-\int_{\Gamma} H\left(\theta, \zeta^{\prime}\right) \varphi(\theta) d \theta-\left(\chi_{\varepsilon} H_{r, \zeta^{\prime}}\right)[\varphi]
$$

where $\varphi \in \mathcal{A}(U)$ and $U \subset \mathbb{C}$ is a compact set such that $\Gamma \cup K_{\varepsilon}^{r} \subset \operatorname{Int} U$. Then it follows from (ii) and (iv) of Definition 1 that

$$
\left|\Xi_{r, a^{\prime}+i b^{\prime}}[\varphi]\right| \leq C \sup _{\theta \in U}|\varphi(\theta)|\left(1+\left\|b^{\prime}\right\|\right)^{\varrho(r)} \quad \text { for } b^{\prime} \in \mathbb{R}^{n-1}
$$

locally uniformly in $a^{\prime} \in \mathbb{R}^{n-1}$. It is also clear that for any $\varphi \in \mathcal{A}\left(\Lambda_{r}\right)$ the function $\mathbb{C}^{n-1} \ni \zeta^{\prime} \mapsto \Xi_{r, \zeta^{\prime}}[\varphi]$ is the Mellin transform of a Mellin distribution in $M^{\prime}\left(\left(0, \tilde{t}^{\prime}\right]\right)$ with support in $\left[\tilde{t}^{\prime}, \tilde{t}^{\prime}\right]$.

As in Section 4, let

$$
\Psi_{\zeta^{\prime}}^{G}\left(\zeta_{1}\right)=\frac{1}{2 \pi i} \Xi_{r, \zeta^{\prime}}\left[G\left(\zeta_{1}-\theta\right)\right] \quad \text { for } \zeta_{1} \in \mathbb{C} \backslash \Lambda_{r}, \zeta^{\prime} \in \mathbb{C}^{n-1},
$$

where $G$ is a modified Cauchy kernel and satisfies (i)-(iv) of Section 3 with $t_{0}>\tilde{t}_{1}$. Again it follows from Definition 1(ii), (iv) that for any open neighbourhood $W$ of $\Lambda_{r}$ we have

$$
\left|\Psi_{a^{\prime}+i b^{\prime}}^{G}\left(a_{1}+i b_{1}\right)\right| \leq C(1+\|b\|)^{\varrho(r)} \quad \text { for } a_{1}+i b_{1} \in \mathbb{C} \backslash W, b^{\prime} \in \mathbb{R}^{n-1}
$$

where $C=C(W, a)$ is locally bounded for $a \in \mathbb{R}^{n}$.

Since $H$ is the Mellin transform of $u \circ S$ it follows that (see [9]) for any $t>\tilde{t}$ and $a<0$ small enough

$$
|H(a+i b)| \leq C t^{-a}(1+\|b\|)^{m} \quad \text { for } b \in \mathbb{R}^{n}
$$

for some constants $C, m$. This together with Definition 1(ii) shows that condition (4) holds. Thus by Theorem 2 (we take $t_{1}<t_{0}$ )

$$
H(\zeta)=\mathcal{C}_{\zeta^{\prime}}^{-}\left(\zeta_{1}\right)+\Psi_{\zeta^{\prime}}^{G}\left(\zeta_{1}\right) \quad \text { for } \zeta_{1} \in \Omega^{1}, \zeta^{\prime} \in \mathbb{C}^{n-1}
$$

where $\mathcal{C}_{\zeta^{\prime}}^{-}\left(\zeta_{1}\right)$ is given by $(6)$. In view of Lemma 3 we have

$$
\mathcal{M}^{1}\left(\Xi_{r, \zeta^{\prime}}\left[y_{1}^{\theta} \chi\left(y_{1}\right)\right]\right)\left(\zeta_{1}\right)=2 \pi i \Psi_{\zeta^{\prime}}^{G}\left(\zeta_{1}\right)
$$

where $\mathcal{M}^{1}$ is the partial Mellin transform in the first variable. Applying to (10) the inverse Mellin transformation $\left(\mathcal{M}^{1}\right)^{-1}$ we find in view of (11)

$$
\left(\mathcal{M}^{1}\right)^{-1} H\left(\cdot, \zeta^{\prime}\right)\left(y_{1}\right)=\frac{1}{2 \pi i} \Xi_{r, \zeta^{\prime}}\left[y_{1}^{\theta} \chi\right]+\left(\mathcal{M}^{1}\right)^{-1} \mathcal{C}_{\zeta^{\prime}}^{-}\left(y_{1}\right) .
$$


Now applying $\left(\mathcal{M}^{\prime}\right)^{-1}$ to both sides of (12) we get (7) with

$$
T_{r}=\frac{1}{2 \pi i}\left(\mathcal{M}^{\prime}\right)^{-1}\left(\Xi_{r, \cdot}\right) \quad \text { and } \quad R_{r} \circ S=\left(\mathcal{M}^{\prime}\right)^{-1}\left(\mathcal{C}^{-}\left(y_{1}\right)\right)
$$

Formula (8) implies that for any $\varphi \in \mathcal{A}\left(\Lambda_{r}\right), T_{r}[\varphi] \in M\left(\mathbb{C}^{n-1} ; \varrho(r)\right)$ and the assignment $\mathcal{A}\left(\Lambda_{r}\right) \ni \varphi \mapsto T_{r}[\varphi] \in M\left(\mathbb{C}^{n-1} ; \varrho(r)\right)$ is continuous. Concerning $R_{r}$, observe that clearly

$$
\mathcal{M}\left(R_{r} \circ S\right)=\mathcal{C}^{-} \in \mathcal{O}\left(\Omega_{r}^{1} \times \mathbb{C}^{n-1}\right), \quad \Omega_{r}^{1} \stackrel{\text { def }}{=} \Omega^{1} \cup\left\{\operatorname{Re} \zeta_{1}<r\right\},
$$

and in view of (10), Definition $1($ ii) and (9)

(13) $\left|\mathcal{M}\left(R_{r} \circ S\right)(a+i b)\right| \leq C(1+\|b\|)^{\tilde{\varrho}\left(a_{1}\right)} \quad$ for $a+i b \in(\mathbb{C} \backslash W) \times \mathbb{C}^{n-1}$

where $W$ is an open neighbourhood of $\Omega_{r}^{1}$ and $\widetilde{\varrho}\left(a_{1}\right)=\max \left(\varrho\left(a_{1}\right), \varrho(r)\right)$. This shows that $R_{r}$ is in the desired space.

Conversely, suppose that the decomposition (7) holds for some $T_{r}$ and $R_{r}$ as in the statement of the theorem. Then (13) holds locally uniformly with respect to $a \in \operatorname{Re} \Omega_{r}^{1} \times \mathbb{R}^{n-1}$. We construct a holomorphic family of extensions to $B(r+$ $i \mathbb{R}) \cap S^{\prime}\left((r+i \mathbb{R}) \backslash K^{r}\right)$ of the function

$$
(r+i \mathbb{R}) \backslash K^{r} \ni \zeta_{1} \mapsto H^{1}\left(\zeta_{1}, \zeta^{\prime}\right)=\mathcal{M}\left(R_{r} \circ S\right)\left(\zeta_{1}, \zeta^{\prime}\right)
$$

for $\zeta^{\prime} \in \mathbb{C}^{n-1}$ as follows: Fix $\varepsilon>0$, take a compact $V \subset \mathbb{C}$ such that $K_{\varepsilon}^{r} \subset$ Int $V$, and $\varphi \in \mathcal{A}(V)$. Let $\Gamma_{1}$ be a continuous curve obtained by modifying the line $(r+i \mathbb{R}) \cap V$ inside $K_{\varepsilon}^{r}$ to a curve contained in $V$ and having $K^{r}$ on its right. Choose a complex neighbourhood $W$ of $\mathbb{C} \backslash \Omega_{r}^{1}$ so that $\Gamma_{1} \subset \mathbb{C} \backslash W$. Define

$$
\chi_{\varepsilon} H_{r, \zeta^{\prime}}^{1}[\varphi]=\int_{\Gamma_{1}} \varphi(\theta) \chi_{\varepsilon}(r+i \operatorname{Im} \theta) H^{1}\left(\theta, \zeta^{\prime}\right) d \theta .
$$

Then it follows from (13) that

$$
\left|\chi_{\varepsilon} H_{r, \zeta^{\prime}}^{1}[\varphi]\right| \leq C_{1} \sup _{\theta \in V}|\varphi(\theta)|\left(1+\left\|b^{\prime}\right\|\right)^{\varrho(r)} .
$$

Next, let $T_{r} \in \mathcal{A}^{\prime}\left(\Lambda_{r} ; M\left(\mathbb{C}^{n-1} ; \varrho(r)\right)\right)$. Then by definition for any compact $U \subset \mathbb{C}$ such that $\Lambda_{r} \subset \operatorname{Int} U$ and $\varphi \in \mathcal{A}(U)$

$$
\left|\mathcal{M}^{\prime}\left(T_{r}[\varphi]\right)\left(a^{\prime}+i b^{\prime}\right)\right| \leq C \sup _{\theta \in U}|\varphi(\theta)|\left(1+\left|b^{\prime}\right|\right)^{\varrho(r)} \quad \text { for } b^{\prime} \in \mathbb{R}^{n-1}
$$

with $C=C\left(U, a^{\prime}\right)$ locally bounded in $a^{\prime} \in \mathbb{R}^{n-1}$. Define

$$
H^{2}(\zeta)=\mathcal{M}\left(T_{r}\left[y_{1}^{\theta} \chi\left(y_{1}\right)\right]\right)(\zeta)=\mathcal{M}^{\prime}\left(T_{r}\left[G\left(\zeta_{1}-\theta\right)\right]\right)\left(\zeta^{\prime}\right)
$$

for $\zeta \in\left(\mathbb{C} \backslash \Lambda_{r}\right) \times \mathbb{C}^{n-1}$. Then for any $U$ as above by (14) and property (iii) of $G$ in Section 3

$$
\left|H^{2}(\alpha+i \beta)\right| \leq C_{2} \frac{\left(1+\left\|b^{\prime}\right\|\right)^{\varrho(r)}}{\left(1+\left|b_{1}\right|\right)^{|\varrho(r)|}} \leq C_{2}(1+\|b\|)^{\varrho(r)}
$$

for $a+i b \in(\mathbb{C} \backslash U) \times \mathbb{C}^{n-1}$ with $C_{2}=C_{2}\left(U, a^{\prime}\right)$ locally bounded in $a^{\prime} \in \mathbb{R}^{n-1}$. Let $\varepsilon$ and $V$ be as for $H^{1}$. Let $\Gamma_{2}$ be a continuous curve obtained by modifying 
the line $(r+i \mathbb{R}) \cap V$ inside $K_{\varepsilon}^{r}$ to a curve contained in $V$ and having $K^{r}$ on its left. Choose $U$ so that $\Gamma_{2} \subset \mathbb{C} \backslash U$. For $\varphi \in \mathcal{A}(V)$, define

$$
\chi_{\varepsilon} H_{r, \zeta^{\prime}}^{2}[\varphi]=\int_{\Gamma_{2}} \varphi(\theta) \chi_{\varepsilon}(r+i \operatorname{Im} \theta) H^{2}\left(\theta, \zeta^{\prime}\right) d \theta .
$$

Then from (15)

$$
\left|\chi_{\varepsilon} H_{r, \zeta^{\prime}}^{2}[\varphi]\right| \leq C_{2} \sup _{\theta \in V}|\varphi(\theta)|\left(1+\left\|b^{\prime}\right\|\right)^{\varrho(r)} .
$$

Finally, defining

$$
H_{r, \zeta^{\prime}}= \begin{cases}\chi_{\varepsilon} H_{r, \zeta^{\prime}}^{1}+\chi_{\varepsilon} H_{r, \zeta^{\prime}}^{2} & \text { on }\left\{\zeta_{1}=r+i b: \operatorname{dist}\left(\zeta_{1}, K^{r}\right)<\varepsilon\right\}, \\ H\left(\zeta_{1}, \zeta^{\prime}\right) & \text { for } \zeta_{1} \in(r+i \mathbb{R}) \backslash K^{r},\end{cases}
$$

we get the desired extension satisfying (iii) and (iv) of Definition 1. Consequently, $u \in Z(\Omega ; \max (\varrho, \varrho(r)))$ and since $r$ is arbitrary it follows that $u \in Z(\Omega ; \varrho)$.

Concerning the uniqueness of (7) assume that

$$
u \circ S=\widetilde{T}_{r}\left[y_{1}^{\theta} \chi\left(y_{1}\right)\right]+\widetilde{R}_{r} \circ S
$$

with $\widetilde{T}_{r}, \widetilde{R}_{r}$ having the same properties as $T_{r}$ and $R_{r}$. Then clearly $T_{r}-\widetilde{T}_{r}$ is in $\mathcal{A}^{\prime}\left(\Lambda_{r} ; M\left(\mathbb{C}^{n-1} ; \varrho(r)\right)\right)$ and by subtracting the Mellin transforms $\mathcal{M}^{1}$ of $(7)$ and (16) we get

$$
\left(T_{r}-\widetilde{T}_{r}\right)\left[G\left(\zeta_{1}-\theta\right)\right]=\mathcal{M}^{1}\left(R_{r} \circ S\right)\left(\zeta_{1}\right)-\mathcal{M}^{1}(\widetilde{R} \circ S)\left(\zeta_{1}\right) .
$$

Since the left-hand side is holomorphic on $\mathbb{C} \backslash \Lambda_{r}$ and the right-hand side on $\Omega_{r}^{1}$ it follows that $\left(T_{r}-\widetilde{T}_{r}\right)\left[G\left(\zeta_{1}-\theta\right)\right]$ is holomorphic outside $K_{r}$. But

$$
\Psi^{G}\left(\zeta_{1}\right)=\frac{1}{2 \pi i}\left(T_{r}-\widetilde{T}_{r}\right)\left[G\left(\zeta_{1}-\theta\right)\right]
$$

is a defining function for $T_{r}-\widetilde{T}_{r}$ (see Theorem 1). Thus $T_{r}-\widetilde{T}_{r}$ has carrier in $K_{r}$.

We now consider Theorem 3 in the setting of the spaces $Z_{\mathrm{d}}(\Omega ; s)$ where $\Omega^{1}=\mathbb{C} \backslash \bigcup_{j=1}^{k} L_{j}$. For a fixed $\zeta^{\prime} \in \mathbb{C}^{n-1}$ and every $j=1, \ldots, k$ define $\Xi_{\zeta^{\prime}}^{j}$ as a hyperfunction on the line $\mathbb{R}+i B_{j}$ whose defining function is $H\left(\cdot, \zeta^{\prime}\right)$ considered as a function in a complex vicinity of $\mathbb{R}+i B_{j}$. Since $H$ satisfies condition (iii) of Definition 2 it follows from Theorem B that $\Xi_{\zeta^{\prime}}^{j}$ is in fact a distribution on $\mathbb{R}+i B_{j}$ with support in $L_{j}$ and can be expressed as

$$
\Xi_{\zeta^{\prime}}^{j}=\lim _{\substack{b_{1} \rightarrow \operatorname{Im} B_{j} \\ b_{1}>\operatorname{Im} B_{j}}} H\left(\cdot+i b_{1}, \zeta^{\prime}\right)-\lim _{\substack{b_{1} \rightarrow \operatorname{Im} B_{j} \\ b_{1}<\operatorname{Im} B_{j}}} H\left(\cdot+i b_{1}, \zeta^{\prime}\right)
$$

where the limit is taken in the sense of distributional convergence in $D^{\prime}\left(\mathbb{R}+i B_{j}\right)$. Moreover,

$$
\left|\Xi_{a^{\prime}+i b^{\prime}}^{j}[\varphi]\right| \leq C \sup _{|\alpha| \leq m}\left|\left(\frac{\partial}{\partial a_{1}}\right)^{\alpha} \varphi\right|\left(1+\left\|b^{\prime}\right\|\right)^{s} \quad \text { for } \varphi \in C_{0}^{\infty}\left(\mathbb{R}+i B_{j}\right) .
$$


Define

$$
T^{j}=\frac{1}{2 \pi i}\left(\mathcal{M}^{\prime}\right)^{-1} \Xi^{j}
$$

where $\left(\mathcal{M}^{\prime}\right)^{-1}$ is the inverse Mellin transformation in the variables $\zeta^{\prime}$. Then it follows from the above that

$$
T^{j} \in D^{\prime}\left(L_{j} ; M\left(\mathbb{C}^{n-1} ; s\right)\right) .
$$

Write $L_{r}^{j}=L_{j} \cap\left\{\operatorname{Re} \zeta_{1} \leq r\right\}(r \in \mathbb{R})$ and let $T_{r}^{j}$ be any distribution in $D^{\prime}\left(\mathbb{R}+i B_{j} ; M\left(\mathbb{C}^{n-1} ; s\right)\right)$ with support in $L_{r}^{j}$ which coincides with $T^{j}$ on $B_{j}+\{a \in$ $\mathbb{R}: a<r\}$. Finally, as usual, set

$$
\Omega_{r}=A^{-1}\left(\left\{\operatorname{Re} \zeta_{1}<r\right\} \cup \Omega^{1} \times \mathbb{C}^{n-1}\right) .
$$

Under the above notation we have the following variant of Theorem 3 .

Theorem $3^{\prime}$. Let $u \in Z_{\mathrm{d}}(\Omega ; s)$. Then for any $r \in \mathbb{R}$ there exists $R_{r} \in M\left(\Omega_{r} ; s\right)$ such that

$$
u \circ S=\sum_{j=1}^{k} T_{r}^{j}\left[y_{1}^{\theta} \chi\left(y_{1}\right)\right]+R_{r} \circ S .
$$

Conversely, if for any $r \in \mathbb{R}$ formula (19) holds for some $T_{r}^{j} \in D_{L_{r}^{j}}^{\prime}\left(\mathbb{R}+i B_{j}\right.$; $\left.M\left(\mathbb{C}^{n-1} ; s\right)\right)(j=1, \ldots, k)$ and $R_{r} \in M\left(\left\{\sum_{j=1}^{n} \operatorname{Re} z_{j}<r\right\} ; s\right)$ then $u \in Z_{\mathrm{d}}(\Omega ; s)$.

Moreover, if (19) holds for some $\stackrel{\circ}{r} \in \mathbb{R}$ then it holds for every $r \leq \stackrel{\circ}{r}$.

P r o of. The first part of the theorem follows immediately from Theorem 3. The proof of the converse implication follows from the lemma below:

Lemma 4. Let $T \in D_{K}^{\prime}\left(\mathbb{R} ; M\left(\mathbb{C}^{n-1} ; s\right)\right)$ with $K$ a bounded interval in $\mathbb{R}$ Define

$$
\Psi_{\zeta^{\prime}}^{G}\left(\zeta_{1}\right)=\frac{1}{2 \pi i} \mathcal{M}^{\prime}\left(T\left[G\left(\theta-\zeta_{1}\right)\right]\right)\left(\zeta^{\prime}\right) \quad \text { for } \zeta_{1} \in \mathbb{C} \backslash K, \zeta^{\prime} \in \mathbb{C}^{n-1} .
$$

Then there exist constants $C=C\left(a^{\prime}\right), p=p\left(a^{\prime}\right)$ locally bounded in $a^{\prime} \in \mathbb{R}^{n-1}$ such that

$$
\left|\Psi_{\zeta^{\prime}}^{G}\left(\zeta_{1}\right)\right| \leq C \frac{\left(1+\left\|b^{\prime}\right\|\right)^{s}}{\left|b_{1}\right|^{p}} \quad \text { for } b_{1} \text { close to } 0 \text { and } b^{\prime} \in \mathbb{R}^{n-1}
$$

Proof. By definition, for $\Xi_{\zeta^{\prime}}=\mathcal{M}^{\prime} T\left(\zeta^{\prime}\right) \in D_{K}^{\prime}(\mathbb{R})$ we have

$$
\left|\Xi_{\zeta^{\prime}}[\varphi]\right| \leq \widetilde{C}\left(\sum_{l=0}^{\tilde{p}} \sup _{t \in K}\left|\left(\frac{d}{d t}\right)^{l} \varphi(t)\right|\right)\left(1+\left\|b^{\prime}\right\|\right)^{s} \quad \text { for } \varphi \in C^{\infty}(\mathbb{R})
$$

where $\widetilde{C}=\widetilde{C}\left(a^{\prime}\right), \widetilde{p}=\widetilde{p}\left(a^{\prime}\right)$ are locally bounded. Thus, putting $\varphi(t)=G\left(t-\zeta_{1}\right)$ for $\zeta_{1}=a_{1}+i b_{1}$ with $b_{1} \neq 0$, in view of property (ii) of $G$, we get (20) with $p=\widetilde{p}+1$. 
Returning to the proof of the theorem we note that the final statement can easily be derived from the above lemma and Lemma 1.

Rem arks. 1. Observe that the condition $R_{r} \in M\left(\Omega_{r} ; \max (\varrho, \varrho(r))\right)$ in Theorem 3 can be replaced by a weaker one:

$$
\left|\mathcal{M}\left(R_{r} \circ S\right)(\zeta)\right|<C(1+\|b\|)^{\varrho(r)} \quad \text { for } \zeta \in\left(\left\{\operatorname{Re} \zeta_{1} \leq r\right\} \backslash V\right) \times \mathbb{C}^{n-1},
$$

where $V$ is an open neighbourhood of $K_{r}$ in $\mathbb{C}$ and $C=C(V, a)$ is locally bounded in $a$.

2. If estimates in the supremum norm in Definitions 1 and 2 are replaced by those in $L^{2}$ norm we obtain an analogue of Theorems 3 and $3^{\prime}$ in terms of the weighted Sobolev spaces $S P\left(s, s^{\prime}\right)$ (see [14]).

3. Different variants of the decompositions (7) and (19) can be used to extend the classical concept of differentiability.

Corollary 1. The spaces $Z(\Omega ; \varrho)$ and $Z_{\mathrm{d}}(\Omega ; \varrho)$ are 2 -local, i.e. for any conical cut-off function $\kappa$, if $u \in Z(\Omega ; \varrho)\left(Z_{\mathrm{d}}(\Omega ; \varrho)\right.$, resp.) then $\kappa u \in Z(\Omega ; \varrho)$ $\left(Z_{\mathrm{d}}(\Omega ; \varrho)\right.$, resp. $)$.

This follows from Theorems 3 and $3^{\prime}$ and Theorem 2 of [11].

6. The radial characteristic set $\operatorname{char}_{\alpha} P$ of a polynomial. This section extends the results of Section 3 of [11]. We start with the following property of the classical Cauchy transformation.

Proposition 1. Let $T$ be a distribution in $E^{\prime}(\mathbb{R})$ of order $\widetilde{p}$ with bounded support. Suppose $T$ restricted to an interval $(0, \stackrel{\circ}{b}), \stackrel{\circ}{b}>0$, is a differentiable function such that for $j=0,1$

$$
\left|\left(\frac{\partial}{\partial \gamma}\right)^{j} T(\gamma)\right| \leq \frac{C}{\gamma^{p}} \quad \text { for } \gamma \in(0, \stackrel{\circ}{b})
$$

where $C>0, p \geq 0$ are some constants. Then the (classical) left Cauchy transform

$$
C^{-} T(z)=-\frac{1}{2 \pi} T\left[\frac{1}{z-i \gamma}\right]
$$

defined and holomorphic for $\operatorname{Re} z<0$ extends to a continuous function on the set $\{z \in \mathbb{C}: \operatorname{Re} z \leq 0,0<\operatorname{Im} z<\stackrel{\circ}{b}\}$ and for every $\stackrel{\circ}{\alpha}<0$ there exists $\widetilde{C}>0$ such that

$$
\left|C^{-} T(\alpha+i \beta)\right| \leq \frac{\widetilde{C}}{\beta^{\hat{p}}} \quad \text { for } \stackrel{\circ}{\alpha} \leq \alpha<0 \text { and small } \beta>0,
$$

where $\widehat{p}=\max (p, \widetilde{p}+1)$.

Proof. Let $\psi \in C_{0}^{\infty}(\mathbb{R})$ be such that $\psi \equiv 0$ on $\mathbb{R} \backslash(-2 / 3,2 / 3),|\psi| \leq 1, \psi \equiv 1$ on $(-1 / 2,1 / 2)$ and $\psi(-\gamma)=\psi(\gamma)$ for $\gamma \in \mathbb{R}$. Let $\beta>0$ be so small that $T$ is a 
differentiable function $T(\gamma)$ on $\operatorname{supp} \psi_{\beta}$ where $\psi_{\beta}(\gamma)=\psi((\gamma-\beta) / \beta)$. Write

$$
T\left[\frac{1}{z-\gamma}\right]=I_{1}(z)+I_{2}(z)
$$

where

$$
I_{1}(\alpha+i \beta)=\int_{\mathbb{R}} \frac{T(\gamma) \psi_{\beta}(\gamma)}{\alpha+i(\beta-\gamma)} d \gamma, \quad I_{2}(\alpha+i \beta)=\left(1-\psi_{\beta}\right) T\left[\frac{1}{\alpha+i(\beta-\gamma)}\right]
$$

We first estimate $I_{1}$. To this end, for $\gamma \in \mathbb{R}$ we write

$$
\psi_{\beta}(\gamma) T(\gamma)=\psi_{\beta}(\gamma) T(\beta)+\psi_{\beta}(\gamma)(\gamma-\beta) \int_{0}^{1} T^{\prime}(\beta+\theta(\gamma-\beta)) d \theta
$$

Observe that since $\psi$ is even we have for $\alpha<0$

$$
\begin{aligned}
\int_{\mathbb{R}} \frac{\psi_{\beta}(\gamma)}{\alpha+i(\beta-\gamma)} d \gamma & =\int_{\mathbb{R}} \frac{\psi(\delta / \beta)}{\alpha+i \delta} d \delta=\int_{\mathbb{R}} \frac{(\alpha-i \delta) \psi(\delta / \beta)}{\alpha^{2}+\delta^{2}} d \delta \\
& =\alpha \int_{\mathbb{R}} \frac{\psi(\delta / \beta)}{\alpha^{2}+\delta^{2}} d \delta=\int_{\mathbb{R}} \frac{\psi(\alpha \varrho / \beta)}{1+\varrho^{2}} d \varrho .
\end{aligned}
$$

For fixed $\beta$ and $\alpha \rightarrow 0_{-}$, the last integral tends to $\int \frac{1}{1+\varrho^{2}} d \varrho=\pi$. Since $|\psi(\alpha \varrho / \beta)|$ $\leq 1$, together with $(24)$ for $j=0$ this gives

$$
\left|T(\beta) \int_{\mathbb{R}} \frac{\psi_{\beta}(\gamma)}{\alpha+i(\beta-\gamma)} d \gamma\right| \leq C_{1} \frac{1}{|\beta|^{p}} \quad \text { for } \stackrel{\circ}{\alpha} \leq \alpha<0 \text { and small } \beta>0 .
$$

Next for $\stackrel{\circ}{\alpha} \leq \alpha<0$ and small $\beta>0$ we have in view of (24) for $j=1$

$$
\begin{aligned}
\mid \int_{\mathbb{R}} & \frac{(\gamma-\beta) \psi((\gamma-\beta) / \beta) \int_{0}^{1} T^{\prime}(\beta+\theta(\gamma-\beta)) d \theta}{\alpha+i(\beta-\gamma)} d \gamma \mid \\
& \leq C_{1} \int_{\mathbb{R}} \int_{0}^{1}\left|T^{\prime}(\beta+\theta(\gamma-\beta))\right| d \theta \psi((\gamma-\beta) / \beta) d \gamma \\
& \leq C_{2} \int_{\mathbb{R}} \int_{0}^{1} \frac{\psi((\gamma-\beta) / \beta)}{|\beta+\theta(\gamma-\beta)|^{p}} d \theta d \gamma \leq C_{2} \int_{0}^{1} \int_{-2 \beta / 3}^{2 \beta / 3} \frac{d \delta d \theta}{|\beta+\theta \delta|^{p}} \leq C_{3} \frac{1}{|\beta|^{p}} .
\end{aligned}
$$

Now, consider $I_{2}(z)$ for $\stackrel{\circ}{\alpha} \leq \alpha<0$ and $\beta$ small. Let $\operatorname{supp} T \subset K$, a bounded interval in $\mathbb{R}$. Since $\operatorname{supp}\left(1-\psi_{\beta}\right) T \subset K \backslash(\beta / 2,3 \beta / 2)$ and $T$ is of order $\widetilde{p}$, we have

$$
\begin{aligned}
\left|I_{2}(\alpha+i \beta)\right| & \leq \sum_{j=0}^{\tilde{p}} \sup _{\gamma \in K \backslash(\beta / 2,3 \beta / 2)}\left|\frac{d^{j}}{d \gamma^{j}} \frac{1}{\alpha+i(\beta-\gamma)}\right| \\
& \leq C_{1} \sup _{\gamma \in K \backslash(\beta / 2,3 \beta / 2)} \frac{1}{|\beta-\gamma|^{\tilde{p}+1}} \leq C_{2} \frac{1}{|\beta|^{\tilde{p}+1}} .
\end{aligned}
$$

We shall also need the following parameter version of Proposition 1: 
COROLlaRY 2. Let $\mathbb{C}^{n-1} \ni \zeta^{\prime} \mapsto T_{\zeta^{\prime}} \in E^{\prime}(\mathbb{R})$ be a distribution-valued holomorphic function which is rapidly decreasing as a function of $\operatorname{Im} \zeta^{\prime}$, locally uniformly in $\operatorname{Re} \zeta^{\prime}$. Suppose that $T_{\zeta^{\prime}}$ restricted to an interval $(0, \stackrel{\circ}{b}), \stackrel{\circ}{b}>0$, is a differentiable function $T_{\zeta^{\prime}}\left(\gamma_{1}\right)$ for $\zeta^{\prime} \in \mathbb{C}^{n-1}$, and for $j=0,1$ and some $l \in \mathbb{N}_{0}$

$$
\left\|\frac{\partial^{j}}{\partial \gamma_{1}^{j}} T_{a^{\prime}+i \cdot}\left(\gamma_{1}\right)\right\|_{l} \leq \frac{C_{l}}{\gamma_{1}^{p}}, \quad \gamma \in(0, \stackrel{\circ}{b}),
$$

locally uniformly with respect to $a^{\prime} \in \mathbb{R}^{n-1}$, where

$$
\left\|\left|\sigma \|_{l}=\sup _{x \in \mathbb{R}^{n-1}}\langle x\rangle^{l} \sum_{|\alpha| \leq l}\right| D^{\alpha} \sigma(x) \mid\right.
$$

for $\sigma \in S\left(\mathbb{R}^{n-1}\right)$. Then for $\stackrel{\circ}{a}_{1} \leq a_{1}<0$ and small $b_{1}>0$

$$
\left\|C^{-} T_{a^{\prime}+i \cdot}\left(a_{1}+i b_{1}\right)\right\|_{l} \leq \frac{\widetilde{C}_{l}}{\gamma_{1}^{\hat{p}}}
$$

locally uniformly in $a^{\prime} \in \mathbb{R}^{n-1}$, where $\widehat{p}=\max (p, 1+\widetilde{p}), \widetilde{p}=\sup _{\zeta^{\prime} \in \mathbb{C}^{n-1}}$ ord $T_{\zeta^{\prime}}$ and

$$
C^{-} T_{\zeta^{\prime}}\left(\zeta_{1}\right)=-\frac{1}{2 \pi} T_{\zeta^{\prime}}\left[\frac{1}{\zeta_{1}-i \gamma}\right] \quad \text { for } \zeta^{\prime} \in \mathbb{C}^{n-1}, \operatorname{Re} \zeta_{1}<0 .
$$

The corollary follows easily from Proposition 1 since $C^{-}$commutes with differentiations in the variable $b^{\prime}$.

Before passing to the definition of the radial characteristic set of a polynomial we recall some properties of the Mellin transforms of conical cut-off functions and of the related Cauchy transforms. Details and proofs are found in [11].

Proposition A. Let $\kappa$ be a conical cut-off function of Definition B. Define

$$
\begin{gathered}
\kappa^{\prime}\left(y^{\prime}\right)=\widetilde{\kappa}\left(1, y^{\prime}\right), \\
K^{\prime}\left(\zeta^{\prime}\right)=\mathcal{M}^{\prime}\left(\kappa^{\prime}\right)\left(\zeta^{\prime}\right) \quad \text { for } \zeta^{\prime} \in \mathbb{C}^{n-1}, \\
K(\zeta)=(\mathcal{M} \kappa) \circ A^{-1}(\zeta) \quad \text { for } \zeta \in(\mathbb{C} \backslash\{0\}) \times \mathbb{C}^{n-1} .
\end{gathered}
$$

Then

(i) $K \in \mathcal{O}\left((\mathbb{C} \backslash\{0\}) \times \mathbb{C}^{n-1}\right)$,

(ii) for every $a \in \mathbb{R}$ the function $\mathbb{R}^{n} \ni b \mapsto\left(a_{1}+i b_{1}\right) K(a+i b)$ is in $S\left(\mathbb{R}^{n}\right)$ locally uniformly with respect to $a \in \mathbb{R}^{n}$,

(iii) $K(\zeta)=-K^{\prime}\left(\zeta^{\prime}\right) / \zeta_{1}+\widetilde{K}(\zeta)$ with $\widetilde{K} \in \mathcal{O}\left(\mathbb{C}^{n}\right)$.

Moreover, $\kappa^{\prime} \in C_{0}^{\infty}\left(\mathbb{R}_{+}^{n-1}\right)$ and

(i') $K^{\prime} \in \mathcal{O}\left(\mathbb{C}^{n-1}\right)$,

(ii') for every $a^{\prime} \in \mathbb{R}^{n-1}$ the function $\mathbb{R}^{n-1} \ni b^{\prime} \mapsto K^{\prime}\left(a^{\prime}+i b^{\prime}\right)$ is in $S\left(\mathbb{R}^{n-1}\right)$ locally uniformly with respect to $a^{\prime} \in \mathbb{R}^{n-1}$.

Theorem C. Let $T \in S^{\prime}\left(\mathbb{R}^{n}\right)$ and fix $\stackrel{*}{a} \in \mathbb{R}^{n}$. Fix a conical cut-off function $\kappa$ as in Definition B, and let $\kappa^{\prime}, K^{\prime}$ and $K$ be defined by (22), (23) and (24) 
respectively. Define

$$
\widetilde{\mathcal{C}}^{ \pm}(\zeta)=\widetilde{\mathcal{C}}^{ \pm} T(\zeta)=\frac{1}{(2 \pi)^{n}} T[K(\zeta-\stackrel{*}{a}-i \gamma)] \quad \text { for } \pm \operatorname{Re} \zeta_{1}> \pm \stackrel{*}{a}{ }_{1}, \quad \zeta^{\prime} \in \mathbb{C}^{n-1},
$$

and

$$
\begin{aligned}
\widetilde{\mathcal{C}}_{a_{1}}^{\prime}\left(\zeta^{\prime}\right) & =\left(\widetilde{\mathcal{C}}^{\prime} T\right)_{a_{1}}\left(\zeta^{\prime}\right) \\
& =\frac{1}{(2 \pi)^{n-1}} T\left[K^{\prime}\left(\zeta^{\prime}-\stackrel{*}{\prime}^{\prime}-i \gamma^{\prime}\right)\right] \in S^{\prime}(\mathbb{R}) \quad \text { for } \zeta^{\prime} \in \mathbb{C}^{n-1}
\end{aligned}
$$

(in (25), $T$ is regarded as an element of $S^{\prime}\left(\mathbb{R}^{n-1} ; S^{\prime}(\mathbb{R})\right)$ under the canonical isomorphism $\left.S^{\prime}\left(\mathbb{R}^{n}\right) \simeq S^{\prime}\left(\mathbb{R}^{n-1} ; S^{\prime}(\mathbb{R})\right)\right)$. Then

$$
\widetilde{\mathcal{C}}^{ \pm} T \in \mathcal{O}\left(\left\{ \pm \operatorname{Re} \zeta_{1}> \pm{\stackrel{*}{a_{1}}}\right\} \times \mathbb{C}^{n-1}\right), \quad\left(\widetilde{\mathcal{C}}^{\prime} T\right)_{a_{1}} \in \mathcal{O}\left(\mathbb{C}^{n-1} ; S^{\prime}(\mathbb{R})\right)
$$

and in the sense of convergence in $S^{\prime}\left(\mathbb{R}^{n}\right)$

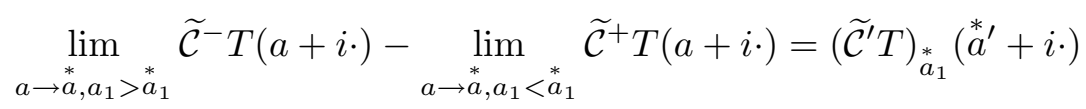

(here $\left(\widetilde{\mathcal{C}^{\prime}} T\right)_{a_{1}}\left({ }^{*}{ }^{\prime}+i \cdot\right) \in S^{\prime}\left(\mathbb{R}^{n-1} ; S^{\prime}(\mathbb{R})\right)$ is regarded as an element of $\left.S^{\prime}\left(\mathbb{R}^{n}\right)\right)$.

Corollary A. Let $H$ be a function holomorphic on an open set $U \subset \mathbb{C}^{n}$. Fix $\stackrel{*}{a} \in \mathbb{R}^{n}$ and suppose that the function $b \mapsto H(\stackrel{*}{a}+i b)$, defined for $b \in \mathbb{R}^{n}$ such that $\stackrel{*}{a}+i b \in U$, extends to a distribution in $S^{\prime}\left(\mathbb{R}^{n}\right)$ which we denote by $H_{*}$. Further, suppose that there exists an open set $U^{1} \subset \mathbb{C}$ such that for every $\zeta_{1} \in U^{1}$ the function $b^{\prime} \mapsto H_{\zeta_{1}}\left(a^{\prime}+i b^{\prime}\right)$, defined for $b^{\prime} \in \mathbb{R}^{n-1}$ such that $\left(\zeta_{1},{ }^{*} a^{\prime}+i b^{\prime}\right) \in U$, extends to a distribution $H_{\zeta_{1}, a^{\prime}}$ in $S^{\prime}\left(\mathbb{R}^{n-1}\right)$, and the distribution-valued function

$$
U^{1} \ni \zeta_{1} \mapsto H_{\zeta_{1}, \stackrel{*}{a^{\prime}}} \in S^{\prime}\left(\mathbb{R}^{n-1}\right)
$$

is holomorphic on $U^{1}$. Finally, assume that there exists a regularization $\widetilde{H}_{a_{1}, a^{\prime}} \in$ $S^{\prime}\left(\mathbb{R} ; S^{\prime}\left(\mathbb{R}^{n-1}\right)\right)$ of the function $b_{1} \mapsto H_{a_{1}+i b_{1}, a^{\prime}} \in S^{\prime}\left(\mathbb{R}^{n-1}\right)$, defined for $b_{1} \in \mathbb{R}$ with $\stackrel{*}{a}_{1}+i b_{1} \in U^{1}$, such that $\widetilde{H}_{a_{1}, a^{\prime}}=H_{*}$ under the canonical isomorphism $S^{\prime}\left(\mathbb{R} ; S^{\prime}\left(\mathbb{R}^{n-1}\right)\right) \simeq S^{\prime}\left(\mathbb{R}^{n}\right)$. Then the function

$$
\widetilde{\mathcal{C}}_{\zeta_{1}}^{\prime}\left(\zeta^{\prime}\right)=\frac{1}{(2 \pi)^{n-1}} H_{\zeta_{1}, a^{\prime}}\left[K^{\prime}\left(\zeta^{\prime}-\stackrel{*}{a}^{\prime}-i \gamma^{\prime}\right)\right], \quad\left(\zeta_{1}, \zeta^{\prime}\right) \in U^{1} \times \mathbb{C}^{n-1},
$$

is holomorphic on $U^{1} \times \mathbb{C}^{n-1}$, and for every fixed $\zeta^{\prime} \in \mathbb{C}^{n-1}$ the distribution $\mathcal{C}_{a_{1}}^{\prime}\left(\zeta^{\prime}\right) \in S^{\prime}(\mathbb{R})$ is a regularization of the function

$$
b_{1} \mapsto{\widetilde{\mathcal{C}_{*}^{\prime}}}_{a_{1}+i b_{1}}^{\prime}\left(\zeta^{\prime}\right)
$$


defined for $b_{1} \in \mathbb{R}$ such that $\stackrel{*}{a_{1}}+i b_{1} \in U^{1}$. Moreover, the function

$$
\widetilde{\Psi}(\zeta)= \begin{cases}\widetilde{\mathcal{C}}^{-}(\zeta) & \text { for } \operatorname{Re} \zeta_{1}<\stackrel{*}{a}_{1}, \zeta^{\prime} \in \mathbb{C}^{n-1}, \\ \widetilde{\mathcal{C}}^{+}(\zeta)+\widetilde{\mathcal{C}}_{\zeta_{1}}^{\prime}\left(\zeta^{\prime}\right) & \text { for } \operatorname{Re} \zeta_{1}>a_{1}, \zeta_{1} \in U^{1}, \zeta^{\prime} \in \mathbb{C}^{n-1},\end{cases}
$$

extends to a holomorphic function $\Psi$ on $\left(\left\{\operatorname{Re} \zeta_{1}<\stackrel{*}{a}_{1}\right\} \cup U^{1}\right) \times \mathbb{C}^{n-1}\left(\right.$ here $\widetilde{\mathcal{C}}^{ \pm}(\zeta)=$ $\left(\widetilde{\mathcal{C}}^{ \pm} H_{*}\right)(\zeta)$ as in Theorem $\left.\mathrm{C}\right)$.

We recall the definition of the radial characteristic set $\operatorname{char}_{\alpha} P$ introduced in [11]. Let $P$ be a polynomial in $\mathbb{C}^{n}, n>1$, and $\stackrel{*}{\alpha} \in \mathbb{R}^{n}$. Set $\stackrel{*}{a}=A \stackrel{*}{\alpha}$ and $\mathcal{P}(\zeta)=P \circ A^{-1}(\zeta)$, and let $(1 / \mathcal{P})_{a}$ be a regularization to a distribution in $S^{\prime}\left(\mathbb{R}^{n}\right)$ of the function $b \mapsto 1 / \mathcal{P}(a+i b)$, which always exists by the division theorem.

DefinitionC. Let $\Omega^{1}$ be the largest set (as in Definition A) such that the function

(26) $\widetilde{\mathcal{C}}^{-}(\zeta)=\frac{1}{(2 \pi)^{n}}\left(\frac{1}{\mathcal{P}}\right)_{\underset{*}{*}}[F(a+i \gamma) K(\zeta-\stackrel{*}{a}-i \gamma)] \quad$ for $\operatorname{Re} \zeta_{1}<\stackrel{*}{a_{1}}, \zeta^{\prime} \in \mathbb{C}^{n-1}$

extends to a holomorphic function on $\Omega^{1} \cap \widetilde{\Omega}^{1} \times \mathbb{C}^{n-1}$ for any $F \in \mathcal{O}\left(\widetilde{\Omega}^{1} \times \mathbb{C}^{n-1}\right)$ (with $\widetilde{\Omega}^{1}$ as in Definition A) such that for any open neighbourhood $W$ of $\mathbb{C} \backslash \widetilde{\Omega}^{1}$ there exist constants $C$ and $M$ such that

$$
|F(a+i b)| \leq C\langle b\rangle^{M} \quad \text { for } a+i b \in(\mathbb{C} \backslash W) \times \mathbb{C}^{n-1}
$$

locally uniformly in $a \in \operatorname{Re} \widetilde{\Omega}^{1} \times \mathbb{R}^{n-1}$. We define $\operatorname{char}_{\alpha} P=\mathbb{C}^{n} \backslash A^{-1}\left(\Omega^{1} \times \mathbb{C}^{n-1}\right)$.

In Theorem 4 below we compute $\operatorname{char}_{\alpha}^{*} P$ for a class of polynomials in two complex variables. We start with notation and preliminaries.

Let $P=\sum_{|\varrho| \leq m} b_{\varrho} z^{\varrho}$ be a polynomial in $\mathbb{C}^{2}$ with complex coefficients. We assume that the vector $\gamma=(-1,1)$ is noncharacteristic for $\mathcal{P}$, i.e. $P_{m}(\gamma) \neq 0$ where $P_{m}(z)=\sum_{|\varrho|=m} b_{\varrho} z^{\varrho}$.

Define $\mathcal{P}\left(\zeta_{1}, \zeta_{2}\right)=P\left(\zeta_{1}-\zeta_{2}, \zeta_{2}\right)$ and write $\mathcal{P}\left(\zeta_{1}, \zeta_{2}\right)=a_{m}\left(\zeta_{1}\right) \zeta_{2}^{m}+\ldots+$ $a_{1}\left(\zeta_{1}\right) \zeta_{2}+a_{0}\left(\zeta_{1}\right)$. Observe that $a_{m}\left(\zeta_{1}\right)$ is a constant function

$$
a_{m}=P_{m}((-1,1))=\sum_{|\varrho|=m} b_{\varrho}(-1)^{\varrho_{1}} \neq 0 .
$$

Represent $\mathcal{P}$ as

$$
\mathcal{P}\left(\zeta_{1}, \zeta_{2}\right)=a_{m} \prod_{j=1}^{m}\left(\zeta_{2}-c_{j}\left(\zeta_{1}\right)\right)
$$

where $c_{1}\left(\zeta_{1}\right), \ldots, c_{m}\left(\zeta_{1}\right)$ are the complex roots of $\mathcal{P}$ with $\zeta_{1}$ regarded as a parameter. Define the discriminant of $\mathcal{P}$,

$$
\Delta=\prod_{j<k}\left(c_{j}\left(\zeta_{1}\right)-c_{k}\left(\zeta_{1}\right)\right) .
$$


If $\mathcal{P}$ has no multiple polynomial factors then it follows from Lemmas A.12 and A.13 of [1] that $\Delta$ is a nonzero polynomial in $\zeta_{1}$ and for every $\dot{\zeta}_{1}$ such that $\Delta\left(\dot{\zeta}_{1}\right) \neq 0$ the functions $c_{j}\left(\zeta_{1}\right), j=1, \ldots, m$, are holomorphic in a neighbourhood of $\stackrel{\circ}{\zeta}_{1}$. Further, in a neighbourhood of every point $\stackrel{\circ}{\zeta}_{1}$ such that $\Delta\left(\stackrel{\circ}{\zeta}_{1}\right)=0$ the $c_{j}\left(\zeta_{1}\right)$ have expansions in Puiseux series, i.e. series of the form

$$
c_{j}\left(\zeta_{1}\right)=\sum_{k=l}^{\infty} d_{k}\left(\left(\zeta_{1}-\stackrel{\circ}{\zeta}_{1}\right)^{1 / p}\right)^{k}
$$

for some $p \in \mathbb{N}, l \in \mathbb{Z}$, and since $a_{m}$ is a constant function we can put $l=0$.

Fix $\stackrel{*}{a} \in \mathbb{R}^{2}$. Consider the functions $c_{j}, j=1, \ldots, m$, defined as follows. For $\operatorname{Re} \zeta_{1}>\stackrel{*}{a_{1}}$ close to $\stackrel{*}{a}, c_{j}\left(\zeta_{1}\right)$ are the holomorphic functions satisfying (27) and we choose the following extension of $c_{j}$ to $\left\{\operatorname{Re} \zeta_{1}>\stackrel{*}{a_{1}}\right\}$. Let $\theta_{\nu}(\nu=1, \ldots, N)$ be all points in $\mathbb{C}$ such that $\Delta\left(\theta_{\nu}\right)=0$ and for some $j=1, \ldots, m, c_{j}$ has a Puiseux expansion at $\theta_{\nu}$ with minimal $p>1$ and $\operatorname{Re} \theta_{\nu}>\stackrel{*}{a_{1}}$.

At the points $\theta_{\nu}, c_{j}$ has value $c_{j}\left(\theta_{\nu}\right)$. For $\stackrel{\circ}{\zeta}_{1} \in \mathbb{R}_{+}+\theta_{\nu}$, we define

$$
c_{j}\left(\dot{\zeta}_{1}\right) \stackrel{\text { def }}{=} c_{j}^{+}\left(\stackrel{\circ}{\zeta}_{1}\right)=\lim _{\zeta_{1} \rightarrow \stackrel{\circ}{\zeta}_{1}, \operatorname{Im} \zeta_{1}>\operatorname{Im} \stackrel{\circ}{\zeta}_{1}} c_{j}\left(\zeta_{1}\right) .
$$

We also define $c_{j}^{-}\left(\stackrel{\circ}{\zeta}_{1}\right)=\lim _{\zeta_{1} \rightarrow \stackrel{\circ}{\zeta}_{1}, \operatorname{Im} \zeta_{1}<\operatorname{Im} \stackrel{\circ}{\zeta}_{1}} c_{j}\left(\zeta_{1}\right)$.

Denote by $B_{\mu}(\mu=1, \ldots, M)$ all points in $\mathbb{R}$ such that for some $j=1, \ldots, m$

$$
\operatorname{Re} c_{j}\left(\stackrel{*}{a}_{1}+i B_{\mu}\right)=\stackrel{*}{a}{ }_{2}
$$

For $j$ satisfying $(29)$ we define $\operatorname{sgn}(j ; \mu)=+$ if for $a_{1}>\stackrel{*}{a}{ }_{1}$ close to $\stackrel{*}{a}{ }_{1}$, $b \mapsto \operatorname{Re} c_{j}\left(a_{1}+i b_{1}\right)$ is an increasing function in a neighbourhood of $B_{\mu}$. Otherwise we put $\operatorname{sgn}(j ; \mu)=-$. Finally, for $\zeta_{1} \in \mathbb{C}$ we set

$$
I^{0}\left(B_{\mu}\right)=\{j \text { : formula }(29) \text { holds }\},
$$

$I^{+}\left(\theta_{\nu}\right)=\left\{j: c_{j}\right.$ has a Puiseux expansion at $\theta_{\nu}$ with $p>1$ and

$$
\left.\operatorname{Re} c_{j}\left(\stackrel{*}{a}_{1}+i b_{1}\right)>\stackrel{*}{a_{2}} \text { for } b_{1}>\operatorname{Im} \theta_{\nu} \text { close to } \operatorname{Im} \theta_{\nu}\right\} \text {. }
$$

THEOREM 4. Fix $\stackrel{*}{\alpha} \in \mathbb{R}^{2}$ and let $\stackrel{*}{a}=A \stackrel{*}{\alpha}$. Under the notation and assumptions introduced above, set

$$
\begin{array}{cc}
L_{\mu}=\mathbb{R}+i B_{\mu} & \text { for } \mu=1, \ldots, M \\
\widetilde{L}_{\nu}=\overline{\mathbb{R}}_{+}+\theta_{\nu} & \text { for } \nu=1, \ldots, N \\
\mathbf{L}=\bigcup_{\mu=1}^{M} L_{\mu} \cup \bigcup_{\nu=1}^{N} \widetilde{L}_{\nu}, & \mathbf{L}_{a}=\mathbf{L} \cap\left\{\operatorname{Re} \zeta_{1} \geq \stackrel{*}{a}_{1}\right\} .
\end{array}
$$

Then $\operatorname{char}_{\alpha} P=A^{-1}\left(\mathbf{L}_{*} \times \mathbb{C}\right)$. Moreover, for any $F \in \mathcal{O}\left(\widetilde{\Omega}_{1} \times \mathbb{C}\right)$ such that the function $\mathbb{R}^{\alpha} \ni \gamma_{2} \mapsto F\left(\zeta_{1}, a_{2}+i \gamma_{2}\right)$ is polynomially bounded at $\infty$ locally uniformly in $\zeta_{1}$ and $a_{2}$, the differences of the boundary values of the Cauchy transform 
(26) are distributions $\Xi_{\zeta_{2}}^{\mu} F$ on the lines $L_{\mu} \cap \widetilde{\Omega}^{1}(\mu=1, \ldots, M)$ with support in $L_{\mu} \cap\left\{\operatorname{Re} \zeta_{1} \geq \stackrel{*}{a_{1}}\right\}$, and distributions $\widetilde{\Xi}_{\zeta_{2}}^{\nu} F$ on the lines $\left\{\mathbb{R}+\theta_{\nu}\right\} \cap \widetilde{\Omega}^{1}(\nu=1, \ldots, N)$ with support in $\widetilde{L}_{\nu}$ such that for any $l \in \mathbb{N}$

$$
\left\|\Xi_{a_{2}+i}^{\mu} . F[\varphi]\right\| \|_{l} \leq C_{p} \sup \sum_{|\alpha| \leq p}\left|D^{\alpha} \varphi\right| \quad \text { for } \varphi \in C_{0}^{\infty}\left(L^{\mu} \cap \widetilde{\Omega}^{1}\right)
$$

for some constants $C_{p}=C_{p}\left(a_{2}\right), p=p\left(a_{2}\right)$ locally bounded in $a_{2} \in \mathbb{R}$, and analogous estimates hold for $\widetilde{\Xi}^{\nu}$. Explicitly, we have

$$
\begin{aligned}
& \Xi_{\zeta_{1}}^{\mu} F\left(\zeta_{2}\right) \\
& \quad=\frac{-1}{a_{m}} \sum_{j \in I^{0}\left(B_{\mu}\right)} \operatorname{sgn}(j ; \mu) \frac{K^{\prime}\left(\zeta_{2}-c_{j}^{\operatorname{sgn}(j ; \mu)}\left(\zeta_{1}\right)\right) F\left(\zeta_{1}, c_{j}^{\operatorname{sgn}(j ; \mu)}\left(\zeta_{1}\right)\right)}{\prod_{q=1, q \neq j}^{m}\left(c_{j}^{\operatorname{sgn}(j ; \mu)}\left(\zeta_{1}\right)-c_{q}^{\operatorname{sgn}(j ; \mu)}\left(\zeta_{1}\right)\right)}
\end{aligned}
$$

for $\zeta_{1} \in\left(\mathbb{R}_{+}+\stackrel{*}{a}_{1}+i B_{\mu}\right) \cap \widetilde{\Omega}^{1}, \mu=1, \ldots, M$ with $\Delta\left(\zeta_{1}\right) \neq 0$, and

$$
\begin{aligned}
\widetilde{\Xi}_{\zeta_{2}}^{\nu} F\left(\zeta_{1}\right)=\frac{-1}{a_{m}} \sum_{j \in I^{+}\left(\theta_{\nu}\right)}\left(\frac{K^{\prime}\left(\zeta_{2}-c_{j}^{+}\left(\zeta_{1}\right)\right) F\left(\zeta_{1}, c_{j}^{+}\left(\zeta_{1}\right)\right)}{\prod_{q=1, q \neq j}^{m}\left(c_{j}^{+}\left(\zeta_{1}\right)-c_{q}^{+}\left(\zeta_{1}\right)\right)}\right. \\
\left.-\frac{K^{\prime}\left(\zeta_{2}-c_{j}^{-}\left(\zeta_{1}\right)\right) F\left(\zeta_{1}, c_{j}^{-}\left(\zeta_{1}\right)\right)}{\prod_{q=1, q \neq j}^{m}\left(c_{j}^{-}\left(\zeta_{1}\right)-c_{q}^{-}\left(\zeta_{1}\right)\right)}\right)
\end{aligned}
$$

for $\zeta_{1} \in\left(\mathbb{R}_{+}+\theta_{\nu}\right) \cap \widetilde{\Omega}^{1}, \nu=1, \ldots, N$.

P r o of. In view of Corollary A we are interested in the holomorphic extensions in the variable $\zeta_{1}$ of the function

$$
\widetilde{\mathcal{C}}_{\zeta_{1}}^{\prime}\left(\zeta_{2}\right)=\frac{1}{2 \pi i} \int_{\operatorname{Re} \theta=a_{2}} \frac{K^{\prime}\left(\zeta_{2}-\theta\right) F\left(\zeta_{1}, \theta\right)}{\mathcal{P}\left(\zeta_{1}, \theta\right)} d \theta
$$

defined for $\zeta_{1}=\stackrel{*}{a}_{1}+i b_{1}$ with $b_{1} \neq B_{\mu}$ for $\mu=1, \ldots, M$. Since the function $\mathbb{C} \ni \theta \mapsto K^{\prime}\left(\zeta_{2}-\theta\right) F\left(\zeta_{1}, \theta\right)$ is rapidly decreasing along the imaginary axis locally uniformly in $\zeta_{1}$ and $\zeta_{2}$, it follows that the integral over the line $\operatorname{Re} \theta=\stackrel{*}{a}_{2}$ may be replaced by an integral over $\operatorname{Re} \theta=r$ (for large $r>0$ ) if we add the suitable residuum terms. To this end define for $\zeta_{1} \in \mathbb{C}$

$$
I_{r}^{+}\left(\zeta_{1}\right)=\left\{j: r>\operatorname{Re} c_{j}\left(\stackrel{*}{a}_{1}+i b_{1}\right)>\stackrel{*}{a_{2}} \text { for } b_{1}>\operatorname{Im} \zeta_{1} \text {, close to } \operatorname{Im} \zeta_{1}\right\} .
$$

In view of (27) we have

$$
\begin{aligned}
\widetilde{\mathcal{C}}_{\zeta_{1}}^{\prime}\left(\zeta_{2}\right)=\frac{-1}{a_{m}} \sum_{j \in I_{r}^{+}\left(\zeta_{1}\right)} \frac{K^{\prime}\left(\zeta_{2}-c_{j}\left(\zeta_{1}\right)\right) F\left(\zeta_{1}, c_{j}\left(\zeta_{1}\right)\right)}{\prod_{q=1, q \neq j}^{m}\left(c_{j}\left(\zeta_{1}\right)-c_{q}\left(\zeta_{1}\right)\right)} \\
\quad+\frac{1}{2 \pi i a_{m}} \int_{\operatorname{Re} \theta=r} \frac{K^{\prime}\left(\zeta_{2}-\theta\right) F\left(\zeta_{1}, \theta\right)}{\prod_{j=1}^{m}\left(\theta-c_{j}\left(\zeta_{1}\right)\right)} d \theta
\end{aligned}
$$


The integral in the second summand is holomorphic (as a function of $\zeta_{1}$ for a fixed $\left.\zeta_{2}\right)$ in the set $\widehat{\Omega}_{r}=\left\{\zeta_{1} \in \mathbb{C}: \operatorname{Re} c_{j}\left(\zeta_{1}\right)<r\right.$ for $\left.j=1, \ldots, m\right\}$ and hence (33) gives an extension of $\widetilde{\mathcal{C}}_{\zeta_{1}}^{\prime}\left(\zeta_{2}\right)$ to $\widehat{\Omega}_{r} \cap \widetilde{\Omega}^{1} \cap\left\{\operatorname{Re} \zeta_{1} \geq \stackrel{*}{a}_{1}\right\} \backslash \mathbf{L}$. Since the functions $c_{j}$ are locally bounded we observe (by letting $r \rightarrow+\infty$ ) that all singularities of the extension are contained in the residuum terms, and the computation of the "jumps" of $\widetilde{\mathcal{C}}_{\zeta_{1}}^{\prime}\left(\zeta_{2}\right)$ is now simple. It follows from Corollary A that the holomorphic extension of $\widetilde{\mathcal{C}}^{-}$is given by

(34) $\psi(\zeta)= \begin{cases}\widetilde{\mathcal{C}}^{-}(\zeta) & \text { for } \operatorname{Re} \zeta_{1}<\stackrel{*}{a}, \zeta_{2} \in \mathbb{C}, \\ \widetilde{\mathcal{C}}^{+}(\zeta)+\widetilde{\mathcal{C}}_{\zeta_{1}}^{\prime}\left(\zeta_{2}\right) & \text { for } \operatorname{Re} \zeta_{1}>\stackrel{*}{a}_{1}, \zeta_{1} \in\left(\mathbb{C} \backslash \mathbf{L}_{a}\right) \cap \widetilde{\Omega}^{1}, \zeta_{2} \in \mathbb{C},\end{cases}$

where $\widetilde{\mathcal{C}}^{ \pm}(\zeta)=(2 \pi)^{-2}(1 / \mathcal{P})_{a}[F(\stackrel{*}{a}+i \gamma) K(\zeta-\stackrel{*}{a}-i \gamma)]$ for $\pm \operatorname{Re} \zeta_{1}> \pm \stackrel{*}{a}$. Thus for $\operatorname{Re} \zeta_{1}>\stackrel{*}{a}_{1}$ the jumps of $\psi\left(\cdot, \zeta_{2}\right)$ coincide with those of $\widetilde{\mathcal{C}}_{\zeta_{1}}^{\prime}\left(\zeta_{2}\right)$, which gives formulas (31) and (32).

It remains to prove that $\Xi_{\zeta_{2}}^{\mu} F, \widetilde{\Xi}_{\zeta_{2}}^{\nu} F$ are distributions on the respective lines. To this end we shall modify the function $\psi(\zeta)$ to a function $\widetilde{\psi}(\zeta)$ which has the same jumps as $\psi$ but whose growth properties are easier to investigate. In view of Proposition A(iii) we can write

$$
K(\zeta)=K^{\prime}\left(\zeta_{2}\right) K^{1}\left(\zeta_{1}\right)+\widetilde{K}(\zeta)
$$

where $K^{1}$ is a modified Cauchy kernel in the variable $\zeta_{1}$ and $\widetilde{K} \in \mathcal{O}\left(\mathbb{C}^{2}\right)$ is such that $\widetilde{K}(a+i \cdot) \in S\left(\mathbb{R}^{2}\right)$ locally uniformly in $a \in \mathbb{R}^{2}$. Then we have

$$
\widetilde{\mathcal{C}}^{ \pm}(\zeta)=\psi_{1}(\zeta)+\psi_{2}(\zeta)
$$

where

$$
\begin{array}{ll}
\psi_{1}(\zeta)=\frac{1}{2 \pi} \widetilde{\mathcal{C}}_{a_{1}}^{\prime}\left(\zeta_{2}\right)\left[K^{1}\left(\zeta_{1}-\stackrel{*}{a}-i \cdot\right)\right] & \text { for } \operatorname{Re} \zeta_{1} \neq \stackrel{*}{a}_{1}, \\
\psi_{2}(\zeta)=\frac{1}{(2 \pi)^{2}}\left(\frac{1}{\mathcal{P}}\right)_{a}^{*}[F(\stackrel{*}{\alpha}+i \gamma) \widetilde{K}(\zeta-\stackrel{*}{a}-i \gamma)] & \text { for } \zeta \in \mathbb{C}^{2} .
\end{array}
$$

Since $\psi_{2}$ is an entire function on $\mathbb{C}^{2}$ we are interested in $\psi_{1}$. Let $\chi$ be a $C_{0}^{\infty}(\mathbb{R})$ function which is 1 in a neighbourhood of the points $B_{\mu}(\mu=1, \ldots, M)$ and $\operatorname{Im} \theta_{\nu}$ $(\nu=1, \ldots, N)$. For $\operatorname{Re} \zeta_{1} \neq \stackrel{*}{a}_{1}$ write

$$
\begin{aligned}
& \psi_{3}(\zeta)=\frac{1}{2 \pi} \chi \widetilde{\mathcal{C}}_{a_{1}}^{\prime}\left(\zeta_{2}\right)\left[K^{1}\left(\zeta_{1}-\stackrel{*}{a}_{1}-i \cdot\right)\right], \\
& \psi_{4}(\zeta)=\frac{1}{2 \pi}(1-\chi) \widetilde{\mathcal{C}}_{a_{1}}^{\prime}\left(\zeta_{2}\right)\left[K^{1}\left(\zeta_{1}-\stackrel{*}{a}_{1}-i \cdot\right)\right] .
\end{aligned}
$$

Again $\psi_{4}\left(\cdot, \zeta_{2}\right)$ is holomorphic in complex neighbourhoods of the points $\stackrel{*}{a}_{1}+$ $i B_{\mu}(\mu=1, \ldots, M)$ and $\stackrel{*}{a}_{1}+i \operatorname{Im} \theta_{\nu}(\nu=1, \ldots, N)$ so we are left with $\psi_{3}$. Inserting

$$
K^{1}\left(\zeta_{1}\right)=-1 / \zeta_{1}+\bar{K}\left(\zeta_{1}\right)
$$


where $\bar{K} \in \mathcal{O}(\mathbb{C})$, in the definition of $\psi_{3}$ we find that modulo a holomorphic factor we are led to considering the function

$$
\psi_{5}(\zeta)=\frac{1}{2 \pi} \chi \widetilde{\mathcal{C}}_{a_{1}}^{\prime}\left(\zeta_{2}\right)\left[\frac{1}{\zeta_{1}-\stackrel{*}{a}_{1}-i \gamma_{1}}\right] \quad \text { for } \operatorname{Re} \zeta_{1} \neq \stackrel{*}{a}_{1}
$$

Summing up we find that about the points $\stackrel{*}{a}_{1}+i B_{\mu}, \stackrel{*}{a}{ }_{1}+i \operatorname{Im} \theta_{\nu}$ the function $\psi$ given by (34) has the same jumps as the function

$$
\Phi\left(\zeta_{1}, \zeta_{2}\right)= \begin{cases}\frac{1}{2 \pi} \chi \widetilde{\mathcal{C}}_{a_{1}}^{\prime}\left(\zeta_{2}\right)\left[\frac{1}{\zeta_{1}-\stackrel{*}{a_{1}}-i \gamma_{1}}\right] & \text { for } \operatorname{Re} \zeta_{1}<\stackrel{*}{a_{1}}, \zeta_{2} \in \mathbb{C}, \\ \frac{1}{2 \pi} \chi \widetilde{\mathcal{C}}_{a_{1}}^{\prime}\left(\zeta_{2}\right)\left[\frac{1}{\zeta_{1}-\stackrel{*}{a}{ }_{1}-i \gamma_{1}}\right]+\widetilde{\mathcal{C}}_{\zeta_{1}}^{\prime}\left(\zeta_{2}\right) & \text { for } \operatorname{Re} \zeta_{1}>\stackrel{*}{a_{1}}, \zeta_{2} \in \mathbb{C} .\end{cases}
$$

Next from (33) we find that $\Phi$ may be replaced by

$$
\widetilde{\Phi}\left(\zeta_{1}, \zeta_{2}\right)= \begin{cases}\frac{1}{2 \pi} \int_{\mathbb{R}} \chi\left(\gamma_{1}\right) \frac{E\left(\stackrel{*}{a}+i \gamma_{1}, \zeta_{2}\right)}{\left.\zeta_{1}-\stackrel{*}{a}\right)_{1}-i \gamma_{1}} d \gamma_{1} & \text { for } \operatorname{Re} \zeta_{1}<\stackrel{*}{a_{1}}, \\ \frac{1}{2 \pi} \int_{\mathbb{R}} \chi\left(\gamma_{1}\right) \frac{E\left(\stackrel{*}{a_{1}}+i \gamma_{1}, \zeta_{2}\right)}{\zeta_{1}-\stackrel{*}{a}{ }_{1}-i \gamma_{1}} d \gamma_{1}+E\left(\zeta_{1}, \zeta_{2}\right) & \text { for } \operatorname{Re} \zeta_{1}>\stackrel{*}{a},\end{cases}
$$

where

$$
E\left(\zeta_{1}, \zeta_{2}\right)=\frac{-1}{a_{m}} \sum_{j \in I^{+}\left(\zeta_{1}\right)} \frac{K^{\prime}\left(\zeta_{2}-c_{j}\left(\zeta_{1}\right)\right) F\left(\zeta_{1}, c_{j}\left(\zeta_{1}\right)\right)}{\prod_{j=1, q \neq j}^{m}\left(c_{j}\left(\zeta_{1}\right)-c_{q}\left(\zeta_{1}\right)\right)}
$$

and

$$
I^{+}\left(\zeta_{1}\right)=\left\{j: \operatorname{Re} c_{j}\left(\stackrel{*}{a}_{1}+i b_{1}\right)>\stackrel{*}{a}_{2} \text { for } b_{1}>\operatorname{Im} \zeta_{1} \text {, close to } \operatorname{Im} \zeta_{1}\right\} .
$$

In view of the properties of $F$ the assertion (30) now follows from Corollary 2 and Theorem B.

R e m ar k. Formulas (31) and (32) demonstrate the occurrence of a "coupled" resurgence effect in the spirit of J. Ecalle [3], [4]. This phenomenon is studied in a forthcoming paper [12].

A remarkable feature of the distributions $\Xi_{\zeta_{2}}^{\mu} F$ and $\widetilde{\Xi}_{\zeta_{2}}^{\nu} F$ is the following

Corollary 4. The distribution-valued holomorphic functions

$$
\mathbb{C} \ni \zeta_{2} \mapsto \Xi_{\zeta_{2}}^{\mu} F, \quad \mathbb{C} \ni \zeta_{2} \mapsto \widetilde{\Xi}_{\zeta_{2}}^{\nu} F
$$

are rapidly decreasing in $\operatorname{Im} \zeta_{2}$, locally uniformly in $\operatorname{Re} \zeta_{2}$ even though the function $\mathbb{C} \ni \zeta_{2} \mapsto F\left(\zeta_{1}, \zeta_{2}\right)$ may grow polynomially in $\operatorname{Im} \zeta_{2}$.

7. 2-dimensional elliptic Fuchsian operators in the spaces $Z(\Omega ; \varrho)$ and $Z_{\mathrm{d}}(\Omega ; \varrho)$. This section provides a refinement of the results of Section 4 of [11]. For the sake of completeness we recall some basic definitions introduced there. 
Let

$$
R=R\left(x_{1}, x_{2}, x_{1} \frac{\partial}{\partial x_{1}}, x_{2} \frac{\partial}{\partial x_{2}}\right)
$$

be a linear partial differential operator of order $m \in \mathbb{N}_{0}$ with smooth coefficients defined in a neighbourhood of zero in $\mathbb{R}^{2}$. Setting $P(z)=R(0, z)$ we write $R$ as

where

$$
R=P\left(x \frac{\partial}{\partial x}\right)-Q\left(x, x \frac{\partial}{\partial x}\right)
$$

$$
Q\left(x, x \frac{\partial}{\partial x}\right)=x_{1} Q^{1}\left(x, x \frac{\partial}{\partial x}\right)+x_{2} Q^{2}\left(x, x \frac{\partial}{\partial x}\right)
$$

and $Q^{1}, Q^{2}$ are differential operators of order $m$. We suppose that $P$ satisfies the following ellipticity condition:

For every $\stackrel{\circ}{\alpha} \in \mathbb{R}^{2}$ there exist $C_{1}<\infty$ and $C_{2}>0$ such that

$$
|P(\stackrel{\circ}{\alpha}+i \beta)|>C_{2}(1+\|\beta\|)^{m} \quad \text { for }\|\beta\|>C_{1} .
$$

Theorem 5 (see [11]). Let $w \in Z(\Omega ; s)\left(w \in Z_{\mathrm{d}}(\Omega ; s)\right.$ with $\Omega^{1}=\mathbb{C} \backslash \bigcup_{j=1}^{m} L_{j}$, resp.) 2-locally at $(0, \delta \stackrel{\circ}{x})$. Then for every $\stackrel{*}{\alpha} \in \mathbb{R}^{2}$ with $\left\{\left(z_{1}, z_{2}\right): \operatorname{Re} z_{1}+\operatorname{Re} z_{2}<\right.$ $\left.\stackrel{*}{\alpha}{ }_{1}+\stackrel{*}{\alpha}_{2}\right\} \subset \Omega$ there exists $u_{\alpha} \in Z\left(\Omega \backslash \bigcup_{j=0}^{\infty}\left\{\operatorname{char}_{\alpha} P+\mathfrak{j}\right\} ; s-m\right)\left(u_{*} \in Z_{\mathrm{d}}(\Omega \backslash\right.$ $\left.\bigcup_{j=0}^{\infty}\left\{\operatorname{char}_{\alpha}^{*} P+\mathfrak{j}\right\} ; s-m\right)$, resp. $)$ 2-locally at $(0, \delta \stackrel{\circ}{x})$ with $\mathfrak{j}=(j, 0, \ldots, 0) \in \mathbb{N}_{0}^{n}$ and $R u_{\alpha}=w$ 2-locally at $(0, \delta \stackrel{\circ}{x})$.

Proof. For $u_{\alpha}$ we take the solution $u \in \mathfrak{M}_{\alpha}^{\prime}$ of the equation

$$
P u=Q(\kappa u)+\kappa w
$$

in $\mathbb{R}_{+}^{2}$, which exists by Theorem 2 of $[6]$ for a suitable cut-off function $\kappa$ at $(0, \delta \stackrel{\circ}{x}$. Then we proceed as in the proof of Theorem 4 of [11]: First we observe that by Theorem 4 of $[11], \kappa u_{\alpha} \in M\left(\Omega \backslash \bigcup_{j=0}^{\infty}\left\{\operatorname{char}_{\alpha} P+\mathfrak{j}\right\} ; s-m\right)$, thus we only have to check that the function $H(\zeta)=\mathcal{M}\left(\kappa u_{\alpha}\right) \circ A^{-1}(\zeta)$ satisfies conditions (iii), (iv) of Definition 1 (condition (iii) of Definition 2, resp.). Define

$$
\Omega_{j}=\left\{z \in \mathbb{C}^{n}: \sum \operatorname{Re} z_{l}<\sum \stackrel{*}{\alpha}_{l}+j\right\} \quad \text { for } j \in \mathbb{N}_{0}
$$

Clearly $M\left(\Omega_{0} ; s-m\right)=Z_{\mathrm{d}}\left(\Omega_{0} ; s-m\right)$. Since $\kappa u_{\alpha} \in M\left(\Omega_{0} ; s-m\right)$ it follows by computing the Mellin transform $\mathcal{M}_{\alpha}$ of (37) that

$$
\mathcal{M}_{\alpha}^{*} u_{\alpha}(\beta)=F(\alpha+i \beta) / P(\alpha+i \beta)
$$

where $H(\zeta)=F \circ A^{-1}(\zeta)$ satisfies (i)-(iv) of Definition 1 on $\widetilde{\Omega}^{1}=\left\{\zeta_{1}<\right.$ $\left.\sum \stackrel{*}{\alpha}_{j}+1\right\} \cap \Omega^{1}$ with $\varrho \equiv s$. Now $\mathcal{M}\left(\kappa u_{\alpha}\right) \circ A^{-1}(\zeta)=\widetilde{\mathcal{C}}^{-}(\zeta)$ and by Corollary $\mathrm{A}$ we are interested in $\widetilde{\mathcal{C}}_{\zeta_{1}}^{\prime}\left(\zeta_{2}\right)$ as in the proof of Theorem 4. Thus we have the decomposition (10). The first term clearly has properties (iii), (iv) of Definition 1 on $\widetilde{\Omega}^{1}$ with $\varrho=-\infty$. To get an estimate of the second term for 
$\zeta_{1} \in \widehat{\Omega}_{r} \cap \widetilde{\Omega}^{1}$ we apply Lemma 2. By induction we prove that (iii) and (iv) hold on any set $\Omega_{j}$.

ExAmple. Let $0 \neq{ }^{*} \in \mathbb{R}^{2}$ and denote by $u_{\alpha} \in \mathfrak{M}_{*}^{\prime}\left(\mathbb{R}^{2}\right)$ the solution of the equation $P u_{\alpha}=\delta_{1}$ on $\mathbb{R}_{+}^{2}$ where $P(x \partial / \partial x)=\left(x_{1}^{\alpha} \partial / \partial x_{1}\right)^{2}+\left(x_{2} \partial / \partial x_{2}\right)^{2}$ such that

$$
\mathcal{M}_{\alpha} u_{\alpha}^{*}(\beta)=\frac{1}{\left(\alpha_{1}^{*}+i \beta_{1}\right)^{2}+\left(\alpha_{2}^{*}+i \beta_{2}\right)^{2}} \in L^{1}\left(\mathbb{R}^{2}\right) .
$$

We have $\mathcal{P}\left(\zeta_{1}, \zeta_{2}\right)=\left(\zeta_{1}-\zeta_{2}\right)^{2}+\zeta_{2}^{2}=2\left(\zeta_{2}-c_{1}\left(\zeta_{1}\right)\right)\left(\zeta_{2}-c_{2}\left(\zeta_{1}\right)\right)$ where

$$
c_{1}\left(\zeta_{1}\right)=\frac{1+i}{2} \zeta_{1}, \quad c_{2}\left(\zeta_{1}\right)=\frac{1-i}{2} \zeta_{1}
$$

and $\Delta=i \zeta_{1}$. Further, from (29) we find

$$
B_{1}=\stackrel{*}{a}_{1}-2 \stackrel{*}{a}_{2}, \quad B_{2}=2 \stackrel{*}{a}_{2}-\stackrel{*}{a}_{1}
$$

and since $c_{1}$ and $c_{2}$ are regular there are no points $\theta_{\nu}$. Define

$$
\begin{aligned}
L_{1} & =\left\{\zeta_{1} \in \mathbb{C}: \zeta_{1}=a_{1}+i\left(\stackrel{*}{a}_{1}-2 \stackrel{*}{a}_{2}\right), a_{1} \geq \stackrel{*}{a_{1}}\right\}, \\
L_{2} & =\left\{\zeta_{1} \in \mathbb{C}: \zeta_{1}=a_{1}+i\left(2{ }^{*} a_{2}-\stackrel{*}{a}_{1}\right), a_{1} \geq \stackrel{*}{a_{1}}\right\}, \\
\mathbf{L} & =L_{1} \cup L_{2} .
\end{aligned}
$$

Then it follows from Theorems 4 and 5 (see also Example 3 of [11]) and Theorem 5 of $[11]$ that

$$
\kappa u_{\alpha} \in Z_{\mathrm{d}}(\Omega ;-\infty)
$$

where $\Omega=A^{-1}((\mathbb{C} \backslash \mathbf{L}) \times \mathbb{C})$ and $\kappa=\varphi \widetilde{\kappa}$ is any proper conical cut-off function. Moreover, by Theorem 4 and Corollary 2 the distributions $\Xi_{\zeta_{2}}^{1}, \Xi_{\zeta_{2}}^{2}$ given by (17) equal

$$
\begin{aligned}
& \Xi_{\zeta_{2}}^{1}[\varphi]=a^{1}\left(\zeta_{2}\right) \delta_{\substack{* \\
\left(a_{1}, a_{1}-2 a_{2}\right)}}[\varphi]+\frac{1}{2 i} \int_{L_{1}} \frac{K^{\prime}\left(\zeta_{2}-\frac{1+i}{2} \zeta_{1}\right)}{\zeta_{1}} \varphi\left(\zeta_{1}\right) d \zeta_{1} \\
& \text { for } \varphi \in C_{0}^{\infty}\left(\mathbb{R}+i B_{1}\right) \text {, } \\
& \Xi_{\zeta_{2}}^{2}[\varphi]=a^{2}\left(\zeta_{2}\right) \delta_{\left(a_{1}, 2 a_{2}-{ }_{a}^{*}\right)}^{*}[\varphi]+\frac{1}{2 i} \int_{L_{2}} \frac{K^{\prime}\left(\zeta_{2}-\frac{1-i}{2} \zeta_{1}\right)}{\zeta_{1}} \varphi\left(\zeta_{1}\right) d \zeta_{1} \\
& \text { for } \varphi \in C_{0}^{\infty}\left(\mathbb{R}+i B_{2}\right)
\end{aligned}
$$

where $K^{\prime}\left(\zeta_{2}\right)=\mathcal{M}^{\prime}\left(\widetilde{\kappa}\left(1, y_{2}\right)\right)\left(\zeta_{2}\right)$ and $a^{\mu}\left(\zeta_{2}\right), \mu=1,2$, are some entire functions which are the Mellin transforms of some smooth functions $\widetilde{T}^{\mu}\left(y_{2}\right)$ vanishing for $y_{2}$ close to zero. Computing the inverse Mellin transforms of $\Xi^{\mu}$ with respect to $\zeta_{2}$ and dividing the results by $2 \pi i$ we find

$$
\begin{aligned}
& T^{1}[\varphi]\left(y_{2}\right)=\frac{1}{2 \pi i} \widetilde{T}^{1}\left(y_{2}\right) \delta_{\left(a_{1}^{*}, a_{1}-2 a_{2}\right)}[\varphi]-\frac{1}{4 \pi} \widetilde{\kappa}\left(1, y_{2}\right) \int_{L_{1}} \frac{y_{2}^{(1+i) \zeta_{1} / 2}}{\zeta_{1}} \varphi\left(\zeta_{1}\right) d \zeta_{1} \\
& \text { for } \varphi \in C_{0}^{\infty}\left(\mathbb{R}+i B_{1}\right) \text {, }
\end{aligned}
$$




$$
\begin{aligned}
& T^{2}[\varphi]\left(y_{2}\right)=\frac{1}{2 \pi i} \widetilde{T}^{2}\left(y_{2}\right) \delta_{\left(a_{1}, 2 \stackrel{*}{a}_{2}-\stackrel{*}{a}_{1}\right)}-\frac{1}{4 \pi} \widetilde{\kappa}\left(1, y_{2}\right) \int_{L_{2}} \frac{y_{2}^{(1-i) \zeta_{1} / 2}}{\zeta_{1}} \varphi\left(\zeta_{1}\right) d \zeta_{1} \\
& \text { for } \varphi \in C_{0}^{\infty}\left(\mathbb{R}+i B_{2}\right) \text {. }
\end{aligned}
$$

R e m a rk. Observe that if $\stackrel{*}{\alpha} \rightarrow 0$ then the lines $L_{1}, L_{2}$ tend towards the halfline $\overline{\mathbb{R}}_{+}$, and the distribution $T^{1}+T^{2}$ for $\alpha_{1}>0$ tends towards the corresponding distribution for $u_{0}=\ln \left(\left(\ln x_{1}\right)^{2}+\left(\ln x_{2}\right)^{2}\right)$. Indeed, we have

$$
\frac{1}{a_{1}}\left(y_{2}^{(1+i) a_{1} / 2}+y_{2}^{(1-i) a_{1} / 2}\right)=\frac{2}{a_{1}} y_{2}^{a_{1} / 2} \cos \left(\frac{a_{1}}{2} \ln y_{2}\right),
$$

which agrees with the explicit formula given in Example 1 of [11].

\section{References}

[1] L. Hörmander, The Analysis of Linear Partial Differential Operators I, Springer, 1985.

[2] H. Komatsu, An introduction to the theory of hyperfunctions, Lecture Notes in Math. 287, Springer, 1973, 1-43.

[3] B. Malgrange, Introduction aux travaux de J. Ecalle, prépublication de l'Institut de Fourier, Université de Grenoble, 20 (1984).

[4] F. Pham, B. Candelpergher et C. Nosmos, Visite aux sources, prépublication de l'Université de Nice, 1989.

[5] Z. Szmydt and B. Ziemian, Multidimensional Mellin transformation and partial differential operators with regular singularity, Bull. Polish Acad. Sci. Math. 35 (1987), 167-180.

[6] - - - Local existence and regularity of solutions of singular elliptic operators on manifolds with corner singularities, J. Differential Equations 83 (1990), 1-25.

[7] - - - The Mellin Transformation and Fuchsian Type Partial Differential Equations, Math. Appl. 56, Kluwer, 1992.

[8] B. Ziemian, Taylor formula for distributions, Dissertationes Math. 264 (1988).

[9] —, The Mellin transformation and multidimensional generalized Taylor expansions of singular functions, J. Fac. Sci. Univ. Tokyo 36 (1989), 263-295.

[10] - , The modified Cauchy transformation with applications to generalized Taylor expansions, Studia Math. 102 (1992), 1-24.

[11] - Elliptic corner operators in spaces with continuous radial asymptotics I, J. Differential Equations, to appear.

[12] - Generalized analytic functions, in preparation.

[13] _ Continuous radial asymptotics for solutions to elliptic Fuchsian equations in 2-dimensions, in: Proc. Symp. Microlocal Analysis, Publ. RIMS, Kyoto 26 (1990), 785-801.

[14] B. Ziemian and H. Kołakowski, Second microlocalization and the Mellin transformation, Publ. RIMS Kyoto Univ. 26 (1990), 785-801. 